\title{
Two dark matter candidates: The case of inert doublet and singlet scalars
}

\author{
G. Bélanger $\odot,{ }^{1}$ A. Mjallal, ${ }^{1}$ and A. Pukhov ${ }^{2}$ \\ ${ }^{1}$ LAPTh, Université Grenoble Alpes, USMB,CNRS, F-74940 Annecy, France \\ ${ }^{2}$ Skobeltsyn Institute of Nuclear Physics, Moscow State University, Moscow 119992, Russia
}

(Received 25 August 2021; accepted 19 January 2022; published 16 February 2022)

\begin{abstract}
We consider a multicomponent dark matter model where the dark sector contains a scalar doublet and a complex scalar singlet. We impose a discrete $Z_{4}$ symmetry to ensure such that the lightest component of the doublet, $\tilde{A}$, and the singlet, $\tilde{S}$, are both stable. Interactions between the dark sectors impact significantly dark matter observables, and they allow, in particular, to significantly relax the direct detection constraints on the model. To determine the parameter space that satisfies relic density and theoretical and collider constraints as well as direct and indirect detection limits, we perform two separate scans. The first includes the full parameter space of the model, while the second is dedicated to scenarios with a compressed inert doublet spectrum. In the first case, we find that the singlet is generally the dominant dark matter component, while in the compressed case the doublet is more likely to be the dominant dark matter component. In both cases, we find that the two dark matter particles can have masses that range from around $m_{h} / 2$ to over the $\mathrm{TeV}$ scale. We emphasize the interplay between cosmological astrophysical and collider constraints and show that a large fraction of the parameter space that escapes current constraints is within the sensitivity reach of future detectors such as XENON-nT, Darwin, or the Cherenkov Telescope Array. Important collider signatures are mostly found in the compressed spectrum case with the possibility of probing the model with searches for heavy stable charged particles and disappearing tracks. We also show that semiannihilation processes such as $\tilde{S} \tilde{S} \rightarrow \tilde{A} Z$ could give the dominant signature in indirect detection searches.
\end{abstract}

DOI: $10.1103 /$ PhysRevD.105.035018

\section{INTRODUCTION}

The hypothesis that a new weakly interacting particle at the electroweak scale could explain dark matter (DM) has been subjected to a host of experimental tests both in astroparticle and particle physics [1-9]. Indeed, one of the most attractive features of these scenarios, beyond their theoretical motivation, is the strong correlation between DM production in the early Universe and DM signatures in direct detection (DD), indirect detection (ID), and at colliders. These experimental searches have in recent years severely restricted the parameter space of typical weakly interacting massive particles (WIMP) models [10,11]. While the searches for WIMPs continue in order to cover as much as possible the large theoretical space of DM models, at the same time many more avenues are being explored for DM candidates [12]. These include extending the range of DM masses from the subGeV [13] to the multi$\mathrm{TeV}$ regions [14] or of interaction strengths from feebly [15-17] to strongly interacting [18-21]. Another possibility

Published by the American Physical Society under the terms of the Creative Commons Attribution 4.0 International license. Further distribution of this work must maintain attribution to the author(s) and the published article's title, journal citation, and DOI. Funded by SCOAP ${ }^{3}$. is to consider a more complex dark sector that features more than one stable particle contributing to DM. The hypothesis of multicomponent DM has been entertained for a long time, the archetype being models with a WIMP DM and another very weak or very light particle (axion, axino, gravitino) [22-25]. Within these models, there are no significant interactions between the DM candidates, and most of the studies of the WIMP phenomenology can be directly applied after rescaling the DM density relevant for astroparticle searches to take into account the fact that the WIMP would contribute only to a fraction of the total DM relic density. Collider signatures of these WIMPs are typically independent of the second component unless the spectrum of the dark sector is compressed and the second component which contributes to DM coannihilation has a typical long-lived signature. Moreover specific searches for feeble and/or very light particles are being pursued, notably the searches for axionlike particles [26,27] of very light DM in beam-dump experiments or of particles that are so weakly coupled that they decay outside the detector [28]. In another class of scenarios, the interactions between the WIMP DM components can be large; thus, they can impact DM production and the relic density prediction. For example, one of the DMs could even be decoupled from the Standard Model (SM) and be thermalized only through its interactions with the second dark sector, a process 
dubbed assisted freeze-out [29]. More generally, interactions between two dark sectors would contribute to DM formation even when both interact with the SM [30,31]. Processes such as semiannihilation [32-34] where two DM annihilate into a DM and a SM particle or processes called dark annihilation or DM conversion where a pair of DM particles annihilate into another pair of DM particles $[35,36]$ as well as the decay of one DM into another component can all play important roles in the early Universe. Examples of simple extensions of the SM that have these features include models with two scalars [30,37-42], two fermions [29,43-47], or two vectors $[48,49]$ as DM candidates and models with a combination of scalar, fermion, and vector DM [50-58] as well as other multicomponent models [59-62]. In such models, the DM components can be stabilized by a discrete symmetry such as $Z_{2} \times Z_{2}$ [63-66] or $Z_{N}$ with $N>4$ [31,67-71] The modifications to DM formation not only impact the value of the relic density but also of various observables such as DM direct detection through DM scattering in a large underground detector and/or specific indirect detection signals from DM annihihilation in the Galaxy, as will be detailed below. Moreover, structure formation and other cosmological simulations were performed within multicomponent DM scenarios [72-74]. One of the advantage of multicomponent DM models is that they can explain observed cosmological and astrophysical anomalies that require DM with different mass scales. Typically, the self-interacting DM that can fit the observations on small cosmological scales is very light [75-77], while DM that can explain the gamma-ray excess in the Fermi-LAT data is rather at the electroweak scale [78,79].

In this paper, we consider a model which contains both a doublet and a singlet of scalars in addition to the SM particles, the inert doublet and singlet model (IDSM). We impose a discrete $Z_{4}$ symmetry and require different charges for the doublet and the singlet [30]. This guarantees the stability of the singlet and of the lightest neutral component of the doublet if its decay to two singlets is kinematically forbidden. Moreover, in the specific case of a very compressed spectrum, there can also be a third DM candidate when the second neutral component of the doublet is almost stable. This model, in the limit where the singlet and doublet decouple, incorporates the cases of the much-studied inert doublet [80-82] and scalar singlet [83-88] dark matter models. Having a second dark matter component contributes to relaxing several of the constraints on the submodels even when imposing that the total relic density completely matches the value determined precisely by PLANCK $[89,90]$. In the scalar singlet model, the coupling that is necessary to provide efficient annihilation of DM in the early Universe is the one that is responsible for DM interactions with nuclei; the two requirements are compatible only for a narrow window when the DM mass is close to half the Higgs mass or for heavy DM above the $\mathrm{TeV}$ scale [84,91-95]. Interactions between the two dark sectors adding processes such as DM conversion and semiannihilation [32-34] mean that a reduced coupling of the singlet can be sufficient to prevent overproduction of DM in the early Universe, thus loosening the direct detection constraints as is detailed later.

The inert doublet model is also subject to strong constraints if the DM mass is below the $W$ mass. Here again, efficient enough annihilation in the early Universe requires either a large coupling to the Higgs or coannihilation with the heavier members of the doublet. The former is constrained by direct detection and by invisible Higgs decays, while the latter by collider constraints from Large Electron-Positron Collider (LEP) on charged Higgs [96]. As soon as the $W W$ channel opens up, annihilation becomes very efficient, and the relic density lies below the measured value unless DM is at the $\mathrm{TeV}$ scale [96-99]. The IDM therefore provides typically only a subcomponent of the total DM. In addition to DD, the model is also constrained by indirect detection [100-103] as well as by LHC searches for monojets, multileptons, and or invisible Higgs [104-111]. The presence of another DM component will not only provide a complete picture for DM but also the interactions with another DM component will alter the phenomenology of the IDM.

Our goal is first to make an up-to-date analysis of the constraints on the Z4IDSM model including theoretical constraints such as perturbativity and vacuum stability as well as experimental constraints at colliders (scalar searches at LEP, electroweak precision, Higgs properties, and searches for new particles at LHC), and in astroparticle searches (direct and indirect detection). At the LHC, the most powerful searches apply in the case of light DM (below $m_{h} / 2$ ) and in the case of a compressed doublet spectrum when searches for disappearing tracks and heavy stable charged particles are relevant. Direct detection searches, in particular, XENON1T, contribute to strongly constrain the model. Current indirect detection searches with photons have more moderate impact because the dominant DM component is typically rather heavy while they constrain mainly the region where DM is at the electroweak scale. Indirect searches with antiprotons allow one to probe the model, although these searches are dependent both on the DM profile and the cosmic ray propagation parameters. After having ascertained the current viability of the model, we then discuss the potential of future searches to further probe the model. Among these, we include prospects for direct detection searches with XENON-nT [112] and DARWIN [113] as well as indirect detection with Cherenkov Telescope Array (CTA) [9]. We also comment on the potential of improved searches for invisible Higgs and for monojets at colliders, although these are of limited interest after taking into account all astrophysical constraints.

The paper is organized as follows. The model is described in Sec. II and dark matter observables in Sec. III. Section IV reviews various constraints on the model, and the results of the parameter scan are presented 
in Sec. V both for the generic case and for the compressed doublet scenario. Section VI contains our conclusions.

\section{THE MODEL}

The model features the gauge symmetry and the fermionic content of the SM, while the scalar sector contains in addition to the SM scalar doublet Higgs $H$ an inert doublet $\tilde{H}^{\prime}$ and a complex singlet $\tilde{S}$. The inert doublet has hypercharge $1 / 2$, and the singlet has zero hypercharge. We assume that all particles of the SM, including $H$, are invariant under a discrete $Z_{4}$ symmetry, while the extra doublet and singlet have different charges. This choice provides the simplest way to have two DM candidates, the singlet and the lightest component of the doublet. The transformation properties of the discrete symmetry correspond to a phase rotation, $\phi \rightarrow \exp ^{\left(i \pi / 2 X_{\phi}\right)} \phi$. There are two possibilities for the $Z_{4}$ charge assignment. The first one corresponds to $X_{S}=2 ; X_{H^{\prime}}=1$, while for the second one, $X_{S}=1 ; X_{H^{\prime}}=2$, and in both cases, $X_{H}=0$. We focus on the latter choice as it offers more possibilities for DM formation; namely, it allows for semiannihilations where two DM particles annihilate into a DM particle and a SM particle [32-34]. With this choice of charge assignment, the first dark sector contains the complex singlet, ${ }^{1}$ while the four particles of the second doublet belong to the second dark sector. All fields that are not invariant under $Z_{4}$ belong to one of the dark sectors and are denoted with a tilde. We assume that the $Z_{4}$ symmetry is not broken; thus, $\tilde{H}^{\prime}$ and $\tilde{S}$ do not couple to fermions. We assume that only $H$ develops a vacuum expectation value that spontaneously breaks $S U(2) \times U(1), \quad\langle H\rangle=v / \sqrt{2}$ with $v=246 \mathrm{GeV}$ and $\left\langle\tilde{H}^{\prime}\right\rangle=0,\langle\tilde{S}\rangle=0$. The scalar potential reads

$$
\begin{aligned}
V_{Z_{4}}= & \lambda_{1}\left(|H|^{2}-\frac{v^{2}}{2}\right)^{2}+\mu_{2}^{2}\left|\tilde{H}^{\prime 2}\right|+\lambda_{2}\left|\tilde{H}^{\prime}\right|^{4}+\mu_{S}^{2}|\tilde{S}|^{2}+\lambda_{S}|\tilde{S}|^{4}+\frac{\lambda_{S}^{\prime}}{2}\left(\tilde{S}^{4}+\tilde{S}^{\dagger 4}\right)+\lambda_{S 1}|\tilde{S}|^{2}|H|^{2}+\lambda_{S 2}|\tilde{S}|^{2}\left|\tilde{H}^{\prime}\right|^{2} \\
& +\lambda_{3}|H|^{2}\left|\tilde{H}^{\prime}\right|^{2}+\lambda_{4}\left(H^{\dagger} \tilde{H}^{\prime}\right)\left(\tilde{H}^{\prime \dagger} H\right)+\frac{\lambda_{5}}{2}\left[\left(H^{\dagger} \tilde{H}^{\prime}\right)^{2}+\left(\tilde{H}^{\prime \dagger} H\right)^{2}\right] \\
& +\frac{\lambda_{S 12}}{2}\left(\tilde{S}^{2} H^{\dagger} \tilde{H}^{\prime}+\tilde{S}^{\dagger 2} \tilde{H}^{\prime \dagger} H\right)+\frac{\lambda_{S 21}}{2}\left(\tilde{S}^{2} \tilde{H}^{\prime \dagger} H+S^{\dagger 2} H^{\dagger} \tilde{H}^{\prime}\right),
\end{aligned}
$$

where the components of the dark doublet are

$$
\tilde{H}^{\prime}=\left(\begin{array}{c}
-i \tilde{H}^{+} \\
\frac{\tilde{H}+i \tilde{A}}{\sqrt{2}}
\end{array}\right),
$$

with masses $M_{H^{ \pm}}, M_{H}, M_{A}$ in an obvious notation. The lightest neutral component of the doublet, either $\tilde{H}$ or $\tilde{A}$, can be one of the DM components. For definiteness in the following, we assume that the pseudoscalar $\tilde{A}$ is the DM component, and similar results are expected for $\tilde{H}$. The complex singlet, $\tilde{S}$, with mass $M_{S}$ is the other DM component. In the following, we denote all fields of the dark sectors with a tilde to distinguish them from standard model fields; thus, we use the notation $\tilde{S}, \tilde{S}^{\dagger}$ for the complex singlet dark matter. The scalar sector of the model has 13 independent parameters. We use the five masses of the scalars, $M_{h}$ for the SM Higgs which we fix at $125 \mathrm{GeV}$, and $M_{H}, M_{A}, M_{H^{ \pm}}, M_{S}$, as well as eight couplings,

$$
\lambda_{2}, \lambda_{3}, \lambda_{S}, \lambda_{S}^{\prime}, \lambda_{S 1}, \lambda_{S 2}, \lambda_{S 12}, \lambda_{S 21} \text {. }
$$

The remaining parameters of the potential can be simply related to these through

\footnotetext{
${ }^{1}$ Note that a mass term that split the real and imaginary components of the singlet is not allowed by the $Z_{4}$ symmetry for this charge assignment.
}

$$
\begin{aligned}
& \lambda_{1}=\frac{M_{h}^{2}}{2 v^{2}} ; \quad \mu_{2}^{2}=M_{H^{ \pm}}^{2}-\lambda_{3} \frac{v^{2}}{2} ; \quad \mu_{S}^{2}=M_{S}^{2}-\lambda_{S 1} \frac{v^{2}}{2} \\
& \lambda_{4}=\frac{M_{H}^{2}+M_{A}^{2}-2 M_{H^{ \pm}}^{2}}{v^{2}} ; \quad \lambda_{5}=\frac{M_{H}^{2}-M_{A}^{2}}{v^{2}}
\end{aligned}
$$

For the doublet, the mass parameters can also be replaced with the mass difference with DM,

$$
\Delta^{+}=M_{H+}-M_{A}, \quad \Delta^{0}=M_{H}-M_{A} .
$$

Among the couplings in Eq. (3), the quartic self-couplings $\lambda_{S}, \lambda_{S}^{\prime}$ for the singlet and $\lambda_{2}$ for the doublet will play little role in DM observables. In the inert doublet model, it is customary to choose as the independent parameter the coupling $\lambda_{H h}=\lambda_{3}+\lambda_{4}+\lambda_{5}$ which determines the $h \tilde{H} \tilde{H}$ coupling, $-v \lambda_{H h}$, or $\lambda_{A h}=\lambda_{3}+\lambda_{4}-\lambda_{5}$ which sets the $h \tilde{A} \tilde{A}$ coupling given by $-v \lambda_{A h}$. These are simply related to our independent parameters,

$$
\begin{aligned}
& \lambda_{H h}=\lambda_{3}-\frac{2}{v^{2}}\left(M_{H^{ \pm}}^{2}-M_{H}^{2}\right) ; \\
& \lambda_{A h}=\lambda_{3}-\frac{2}{v^{2}}\left(M_{H^{ \pm}}^{2}-M_{A}^{2}\right) .
\end{aligned}
$$

Other trilinear couplings that will be relevant for the following analyses are the coupling $h \tilde{H}^{+} \tilde{H}^{-},-v \lambda_{3}$ as well as the couplings involved in semiannihilation, the trilinear 
coupling $\tilde{S} \tilde{S} \tilde{A}, i v\left(\lambda_{S 21}-\lambda_{S 12}\right) / 2$, and the coupling $\tilde{S} \tilde{S} \tilde{H}$, $v\left(\lambda_{S 21}+\lambda_{S 12}\right) / 2$. Note that in order to have two stable DM candidates, the decay of the lightest component of the inert doublet into the singlet must be kinematically forbidden; that is, $M_{A / H}<2 M_{S}$. When this condition is violated, only $\tilde{S}$ is the DM, and the model effectively reduces to the scalar singlet model as is discussed in Sec. V. In this model, the DM phenomenology is similar whether the scalar or the pseudoscalar component of the doublet is the DM candidate; in the following, we choose the pseudoscalar as DM.

We have also included in the model effective vertices for $\tilde{H}^{ \pm} \tilde{A} \pi^{\mp}$ and $\tilde{H}^{ \pm} \tilde{H} \pi^{\mp}$ interactions. These vertices become relevant when the mass splitting between the charged and neutral Higgses is below a few hundred MeVs [114]. In this case the dominant decay mode is $\tilde{H}^{ \pm} \rightarrow \pi^{ \pm} \tilde{A}$, which has a much larger decay width then the one computed for decay into quarks $\tilde{H}^{+} \rightarrow u \bar{d} \tilde{A}$. To this end, following Refs. [96,115], we introduce the nonperturbative $W-\pi$ mixing through the Lagrangian,

$$
\mathcal{L}=\frac{g f_{\pi}}{2 \sqrt{2}} W_{\mu}^{+} \partial^{\mu} \pi^{-}
$$

such that the $\tilde{H}^{+} \tilde{A} \pi^{-}$vertex is defined as

$$
\frac{g^{2} f_{\pi}}{4 \sqrt{2} M_{W}^{2}}\left(p_{H^{+}}-p_{A}\right) \cdot p_{\pi}
$$

where $f_{\pi}=130 \mathrm{MeV}$, and $g$ is the $S U(2)$ coupling constant. For decays that take place on the mass shell, the vertex reduces to $g^{2} f_{\pi} / 4 \sqrt{2}\left(M_{H+}^{2}-M_{A}^{2}\right) / M_{W}^{2}$. This effective vertex is taken into account only if $\Delta^{+}$lies in the range $140 \mathrm{MeV} \leq \Delta^{+} \leq 500 \mathrm{MeV}$. For smaller mass splittings, the charged Higgs decays into leptons and a neutral Higgs. For larger mass splittings, the decay of the charged Higgs is prompt, and the decay width into quarks gives an accurate enough result as is shown in [116].

For completeness, we mention that there is an alternative charge assignment corresponding to $X_{S}=2$ and $X_{H^{\prime}}=1$; however, in this case, semiannihilation processes are not allowed. The phenomenology of this model will be discussed in a separate publication. Finally, we stress that we treat the model at tree level, and we assume that all parameters are input at the electroweak scale. We have estimated the loop corrections to the masses of the scalar sector and found that small mass splittings will be induced even for a completely degenerate tree-level spectrum; typically, the mass split with the DM lies below $\approx 500 \mathrm{MeV}(50 \mathrm{MeV})$ for the charged (neutral) component [117]. For self-consistency with the relic density calculation which is performed at tree level, we therefore chose a complete tree-level treatment, keeping in mind that small mass splittings are justified as arising from one-loop corrections. For a complete discussion of the impact of one-loop corrections on the relic density in the inert doublet model, see Ref. [118-122]. The one-loop corrections to the 3-body decay width of the charged Higgs into DM and light leptons were computed for a few typical points and found to be around the percent level [123], thus justifying a treelevel treatment.

\section{DARK MATTER RELIC DENSITY}

The relic density of DM provides one of the most important constraints on the model after we impose the condition that the total relic density of the two DM candidates matches the value measured by PLANCK [89]. Before discussing in general terms DM production in the early Universe within the $Z_{4}$ model, we first briefly review the main characteristics of DM in the IDM and in the scalar singlet model, which in the limit where the two sectors decouple form the two components of DM in the Z4IDSM model.

In the scalar singlet model, all annihilation channels depend on $\lambda_{S 1}$, the coupling between the scalar singlet and the SM Higgs. Thus, the relic density basically fixes this parameter to $\lambda_{S 1} \approx 0.15\left(M_{S} / 1000 \mathrm{GeV}\right)$ except in the region $M_{S} \approx m_{h} / 2$, where it can be much smaller. However, the same coupling also enters the direct detection cross section. Typically, a coupling large enough to provide sufficient DM annihilation in the early Universe leads to a direct detection cross section in conflict with XENON1T results unless the $\mathrm{DM}$ is above the $\mathrm{TeV}$ scale. A narrow region around $m_{S} \approx m_{h} / 2$ that satisfies DD constraints also remains [84,91-94].

In the IDM, the relic density is driven mostly by gauge couplings and by the DM coupling to the Higgs, $\lambda_{H h}$ or $\lambda_{A h}$ for the scalar or pseudoscalar DM, respectively. Typically, DM is underabundant when annihilation into $W$ pairs are kinematically accessible. An important exception is found when the spectrum is compressed and $M_{\mathrm{DM}} \geq 500 \mathrm{GeV}$. In this case, the annihilation into longitudinal gauge bosons can be suppressed, thus allowing for a relic density, 97] $\Omega h^{2} \approx 0.1$ ]. Below the $W$ threshold, the inert Higgs can account for all of the DM if its coupling to the Higgs is large; this is however constrained from direct detection. In this region, underabundant DM is also found if the mass of the inert Higgs is such that $M_{A} \approx m_{h} / 2$, and the DM annihilation cross section is enhanced by a resonance or for a compressed spectrum which benefits from many coannihilation channels. Note that the spin-independent (SI) direct detection cross section also depends on $\lambda_{H h}\left(\lambda_{A h}\right)$ so that the largest values of this coupling are excluded even when the inert doublet is only a subcomponent of DM.

A model with an inert doublet and singlet helps relax these constraints. In particular, the additional processes participating in the DM singlet annihilation (DM conversion or semiannihilation) mean that lower values of $\lambda_{S 1}$ are allowed, thus avoiding strong direct detection constraints on the singlet DM component. Moreover the same process 


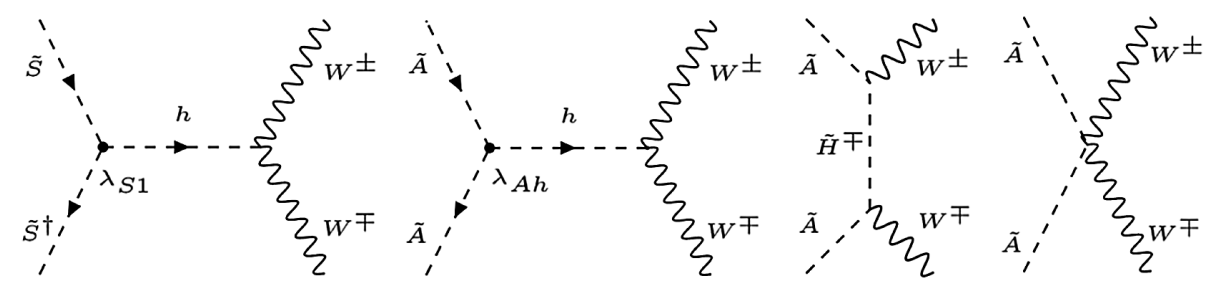

FIG. 1. Sample Feynman diagrams for DM annihilation into gauge bosons; the first diagram on the left contributes to $\left\langle v \sigma^{1100}\right\rangle$, while others contribute to $\left\langle v \sigma^{2200}\right\rangle$.

can also reduce the fraction of the doublet component, hence helping evade direct detection constraints. In the next subsection, we illustrate the impact on the relic density of the interactions between the two dark sectors.

\section{A. Interactions between dark sectors}

In the following, we split the particle content in different sectors according to their $Z_{4}$ charge. The dark sector 1 contains the singlet, and the dark sector 2 contains the doublet, while all SM particles are in sector 0. The two DM candidates are the singlet, $\tilde{S}$, and the lightest neutral component of the doublet which we take to be $\tilde{A}$. The equations for the thermal evolution of the abundances, $Y_{1,2}$, in the two dark sectors read

$$
\begin{aligned}
3 H \frac{d Y_{1}}{d s}= & \left\langle v \sigma^{1100}\right\rangle\left(Y_{1}^{2}-\bar{Y}_{1}^{2}\right)+\left\langle v \sigma^{1120}\right\rangle\left(Y_{1}^{2}-Y_{2} \frac{\bar{Y}_{1}^{2}}{\bar{Y}_{2}}\right) \\
& +\left\langle v \sigma^{1122}\right\rangle\left(Y_{1}^{2}-Y_{2}^{2} \frac{\bar{Y}_{1}^{2}}{\bar{Y}_{2}^{2}}\right), \\
3 H \frac{d Y_{2}}{d s}= & \left\langle v \sigma^{2200}\right\rangle\left(Y_{2}^{2}-\bar{Y}_{2}^{2}\right)-\frac{1}{2}\left\langle v \sigma^{1120}\right\rangle\left(Y_{1}^{2}-Y_{2} \frac{\bar{Y}_{1}^{2}}{\bar{Y}_{2}}\right) \\
& +\left\langle v \sigma^{1210}\right\rangle Y_{1}\left(Y_{2}-\bar{Y}_{2}\right)+\left\langle v \sigma^{2211}\right\rangle\left(Y_{2}^{2}-Y_{1}^{2} \frac{\bar{Y}_{2}^{2}}{\bar{Y}_{1}^{2}}\right) .
\end{aligned}
$$

Here, it is assumed that all particles within a dark sector are in chemical equilibrium. $\bar{Y}_{1,2}$ are the equilibrium abundances in each sector, and $s$ is the entropy density, $H$ the Hubble parameter, and $\left\langle v \sigma^{i j k l}\right\rangle$ the thermally averaged cross sections for all processes of the type $i, j \rightarrow k, l$ involving particles in sectors 0,1 , and 2 . In these equations, there is an implicit summation over all particles that are involved in a given subprocess; e.g., $\left\langle v \sigma^{2200}\right\rangle$ includes all processes involving the annihilation of pairs of $\tilde{H^{ \pm}}, \tilde{H}, \tilde{A}$ into pairs of SM particles. As in single DM models, coannihilation processes involving heavier particles of the dark sector are strongly suppressed. Note that the thermally averaged cross section $v \sigma^{i j k l}$ satisfies the balance equation

$$
\left(1-\frac{1}{2} \delta_{i j}\right) \bar{Y}_{i} \bar{Y}_{j}\left\langle v \sigma^{i j k l}\right\rangle=\left(1-\frac{1}{2} \delta_{k l}\right) \bar{Y}_{k} \bar{Y}_{l}\left\langle v \sigma^{k l i j}\right\rangle .
$$

Figure 1 shows sample diagrams contributing to DM annihilation into SM particles within each sector. The interactions between the two dark sectors leading to DM conversion $\left(v \sigma^{1122}, v \sigma^{2211}\right)$ and the presence of semiannihilation terms $\left(v \sigma^{1120}\right)$ can strongly affect the relic density of each DM candidate, while semiannihilation of the type $v \sigma^{1210}$ will only impact the second DM. Processes which contribute to DM conversion, e.g., $\tilde{S} \tilde{S}^{\dagger} \leftrightarrow \tilde{A} \tilde{A}, \tilde{H} \tilde{H}, \tilde{H}^{+} \tilde{H}^{-}$, are driven either by quartic interactions which depend on the coupling $\lambda_{S 2}$ or by annihilation through the SM Higgs which is proportional to $\lambda_{A h} \lambda_{S 1}$. Samples of Feynman diagrams that contribute to these processes are shown in Fig. 2.

To illustrate the effect of the interactions between the two dark sectors on the relic density of DM, we consider two sample scenarios. For both, we fix $M_{A}=120 \mathrm{GeV}$, $M_{S}=250 \mathrm{GeV}$, and $\lambda_{3}=8 \times 10^{-3}$. We choose three values for the semiannihilation couplings $\lambda_{S 12}=0,0.01$, 1 , while always imposing $\lambda_{S 12}=-\lambda_{S 21}$ to maximize the coupling of the singlet to the pseudoscalar DM, $\tilde{S} \tilde{S} \tilde{A}$. In addition for the first scenario, we take $M_{H^{+}}=M_{H}=$ $125 \mathrm{GeV}$ and $\lambda_{S 1}=10^{-3}$, while $\lambda_{S 2}$ is a free parameter. For the second scenario, we allow a larger mass splitting between the doublet components by choosing $M_{H^{+}}=$ $M_{H}=200 \mathrm{GeV}$, and we fix $\lambda_{S 2}=10^{-5}$ leaving $\lambda_{S 1}$ as a

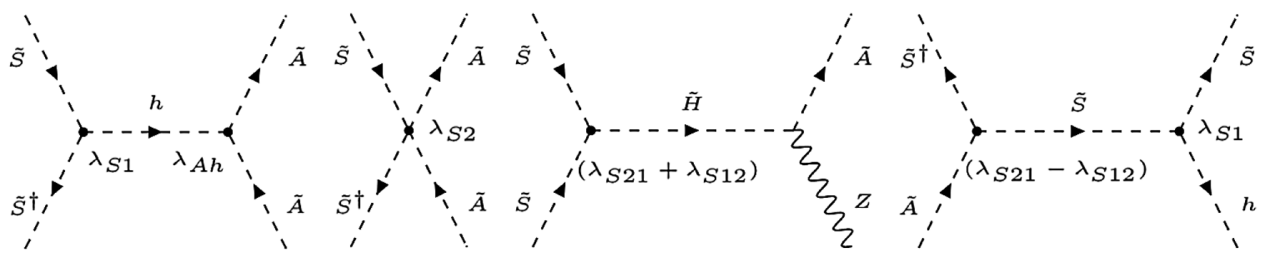

FIG. 2. Sample Feynman diagrams for processes that involve interactions between the two dark sectors; the two diagrams on the left contribute to $\left\langle v \sigma^{1122}\right\rangle$, the third to $\left\langle v \sigma^{1120}\right\rangle$, and the one on the right to $\left\langle v \sigma^{1210}\right\rangle$. 

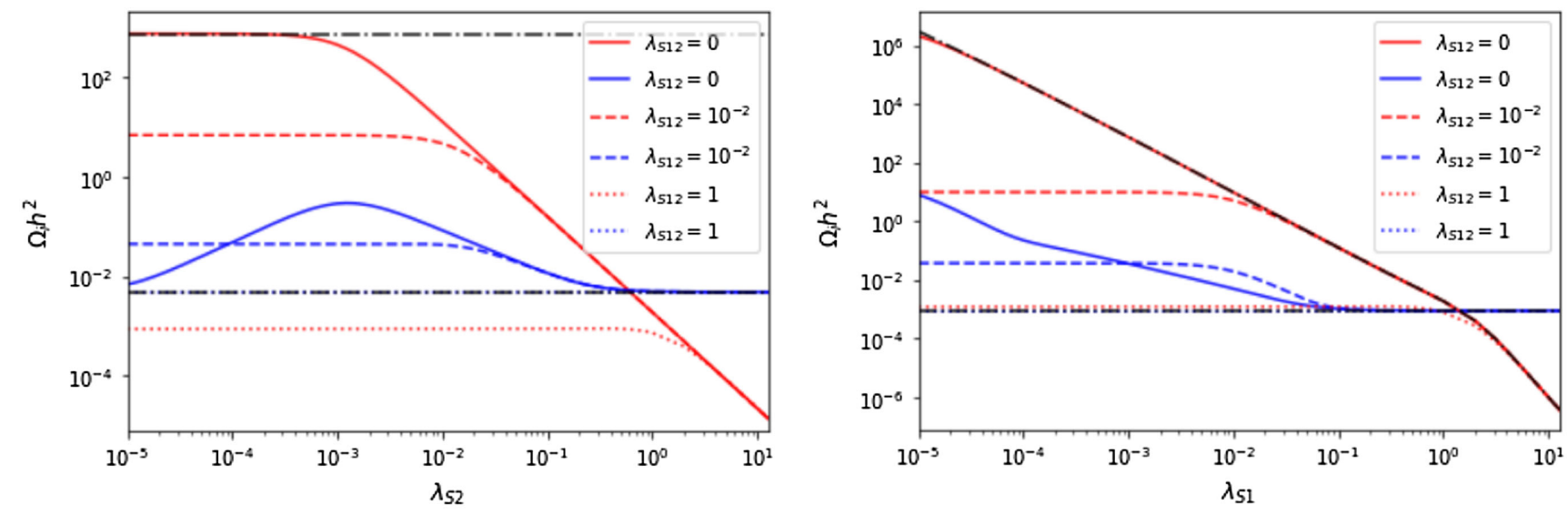

FIG. 3. Dependence of $\Omega_{1} h^{2}$ (red) and $\Omega_{2} h^{2}$ (blue) on $\lambda_{S 2}$ (left) and $\lambda_{S 1}$ (right) for $\lambda_{S 12}=-\lambda_{S 21}=0,0.01,1, M_{A}=120 \mathrm{GeV}$, $M_{S}=250 \mathrm{GeV}$, and $\lambda_{3}=8 \times 10^{-3}$. Other parameters are set to (left) $M_{H^{+}}=M_{H}=125 \mathrm{GeV}, \lambda_{S 1}=10^{-3}$ and (right) $M_{H^{+}}=M_{H}=$ $200 \mathrm{GeV}, \lambda_{S 2}=10^{-5}$. The black lines (dashed-dotted) show $\Omega_{i} h^{2}$ when interactions between the dark sectors are neglected.

free parameter. These two scenarios are used to illustrate the dependence of the relic density on the parameters $\lambda_{S 12}$ and $\lambda_{S 2}$ or $\lambda_{S 1}$.

For the choice of parameters corresponding to the first scenario, we obtain $\lambda_{A h}=-3.2 \times 10^{-2}$. Thus, processes which contribute to DM conversion are driven by the quartic coupling $\lambda_{S 2}$ since the annihilation through the SM Higgs which is proportional to $\lambda_{A h} \lambda_{S 1}$ is suppressed. When we neglect semiannihilations, that is, $\lambda_{S 12}=0$, the relic density of the singlet is very large when both $\lambda_{S 1}, \lambda_{S 2} \ll 1$, see Fig. 3 (left panel). Since $\tilde{S}$ which is heavier freezes-out when $\tilde{A}$ is still in thermal equilibrium, $11 \rightarrow 22$ processes act as additional annihilation channels for $\tilde{S}$; thus, we observe a sharp decrease in $\Omega_{1}$ when $v \sigma^{1122}$ becomes larger than $v \sigma^{1100}$. This occurs for $\lambda_{S 2}>10^{-3}$. The behavior of $\Omega_{2}$ is different. First note that because of gauge interactions with the SM sector, $\Omega_{2}$ is typically quite small. Turning on the interactions between the dark sectors leads to an increase of $\Omega_{2}$ with $\lambda_{S 2}$ due to $11 \rightarrow 22$ processes. Increasing $\lambda_{S 2}$ further, this channel progressively becomes less efficient because the abundance $Y_{1}$ decreases sharply; the turning point is around $\lambda_{S 2}=10^{-3}$. For larger values of $\lambda_{S 2}, \Omega_{2}$ is mostly set by the interactions of the second dark sector with the SM. This is the case even when $\lambda_{S 2}$ becomes $\mathcal{O}(1)$ since the kinematic suppression of the channels $22 \rightarrow 11$ prevents these channels to contribute significantly to $\Omega_{2}$. The impact of semiannihilation processes on $\Omega_{1}$ and $\Omega_{2}$ is similar to that of DM conversion in the sense that an increase of $\lambda_{S 12}$ leads to a decrease of $\Omega_{1}$, while $\Omega_{2}$ increases due to processes such as $\tilde{S} \tilde{S} \rightarrow \tilde{A} h$ or $\tilde{S} h \rightarrow \tilde{S}^{\dagger} \tilde{A}$ as can be seen in Fig. 3 in the limit of small $\lambda_{S 2}$ and for $\lambda_{S 12}=0.01$. For larger values of $\lambda_{S 12}=1$, semiannihilation does not impact $\Omega_{2}$ because the abundance $Y_{1}$ is small.

For the second scenario, we illustrate the dependence on $\lambda_{S 1}$. Recall that this coupling of the singlet to the SM Higgs enters both annihilation processes of the singlet into DM particles as well as into pairs of doublets. However, since $\tilde{S}$ is heavier than $\tilde{A}$, the latter has little impact on the singlet component. Indeed, in the limit where semiannihilation can be neglected $\left(\lambda_{S 12}=0\right)$, we find that $v \sigma^{1100}$ gives the main contribution to $\Omega_{1}$ which decreases when $\lambda_{S 1}$ increases. Moreover, the value of $\Omega_{1} h^{2}$ matches the one obtained neglecting all interactions between the dark sectors, see Fig. 3 (right panel) where the red and dashed-dotted lines are superimposed. Note that when $\lambda_{S 1}$ becomes very large, the annihilation channel $\tilde{S} \tilde{S}^{\dagger} \rightarrow h h$ becomes dominant as the t-channel diagram grows as $\lambda_{S 1}^{4}$, hence the steeper drop in $\Omega_{1} h^{2}$. For the doublet component, the larger mass splitting as compared to the previous case leads to a larger value for $\lambda_{A h}=-0.84$. Thus, we expect efficient annihilation of the doublet into SM particles. Indeed including only those processes, we find $\Omega_{2} h^{2}=1.1 \times 10^{-3}$. DM conversion processes proceed through the s-channel SM Higgs exchange; e.g., $\tilde{S} \tilde{S}^{\dagger} \rightarrow h \rightarrow \tilde{A} \tilde{A}$, since $\lambda_{S 2}$ is small. These processes can significantly increase the abundance of the doublet; for example, for $\lambda_{S 1}=10^{-5}$, we find $\Omega_{2} h^{2}=7.7$ when all processes are included. The impact of DM conversion is not so drastic for larger values of $\lambda_{S 1}$ because it depends on $Y_{1}$ which is suppressed at larger $\lambda_{S 1}$. In fact for large $\lambda_{S 1}$, we expect little dependence of $\Omega_{2}$ on $\lambda_{S 1}$, as seen in Fig. 3 (right panel). As in the previous example, semiannihilation terms decrease $\Omega_{1}$ and have the largest impact at small values of $\lambda_{S 1}$ where $Y_{1}$ is large. Moreover semiannihilation typically also leads to a decrease of $\Omega_{2}$. However, as explained above, semiannihilation processes such as $\tilde{S} \tilde{S} \rightarrow \tilde{A} h$ or $\tilde{S} h \rightarrow \tilde{S}^{\dagger} \tilde{A}$ can lead to an increase in $\Omega_{2}$ if $Y_{1}$ is sizeable; this occurs for moderate values of $\lambda_{S 12}=-\lambda_{S 21}=0.01$.

We also consider the case where the two neutral components of the neutral doublet are nearly degenerate. 
In this case, we solve three components equations, one for the singlet, the second for the doublet components $\tilde{A}, \tilde{H}^{+}$, and the third for $\tilde{H}$. The reason for this is that $\tilde{H}$ can have a small decay width, and it might not remain in chemical equilibrium with $\tilde{A}$ and $\tilde{H}^{+}$, hence the need for a separate equation for the abundance of $\tilde{H}$. Note that we explicitly take into account the decay term corresponding to processes such $\tilde{H} \rightarrow \tilde{A} h$. The equations for the abundances read,

$$
\begin{aligned}
& 3 H \frac{d Y_{1}}{d s}=\left\langle v \sigma^{1100}\right\rangle\left(Y_{1}^{2}-\bar{Y}_{1}^{2}\right)+\sum_{k=2,3}\left[\left\langle v \sigma^{11 k 0}\right\rangle\left(Y_{1}^{2}-Y_{k} \frac{\bar{Y}_{1}^{2}}{\bar{Y}_{k}}\right)+\left\langle v \sigma^{11 k k}\right\rangle\left(Y_{1}^{2}-Y_{k}^{2} \frac{\bar{Y}_{1}^{2}}{\bar{Y}_{k}^{2}}\right)\right]+\left\langle v \sigma^{1123}\right\rangle\left(Y_{1}^{2}-Y_{2} Y_{3} \frac{\bar{Y}_{1}^{2}}{\bar{Y}_{2} \bar{Y}_{3}}\right), \\
& 3 H \frac{d Y_{k}}{d s}=\left\langle v \sigma \sigma^{k k 00}\right\rangle\left(Y_{k}^{2}-\bar{Y}_{k}^{2}\right)+\left\langle v \sigma^{k k 11}\right\rangle\left(Y_{k}^{2}-Y_{1}^{2} \frac{\bar{Y}_{k}^{2}}{\bar{Y}_{1}^{2}}\right)+\left\langle v \sigma^{k k k^{\prime} k^{\prime}}\right\rangle\left(Y_{k}^{2}-Y_{k^{\prime}}^{2} \frac{\bar{Y}_{k}^{2}}{\bar{Y}_{k^{\prime}}^{2}}\right) \\
& +\frac{1}{2}\left\langle v \sigma^{k k 23}\right\rangle\left(Y_{k}^{2}-Y_{2} Y_{3} \frac{\bar{Y}_{k}^{2}}{\bar{Y}_{2} \bar{Y}_{3}}\right)+\left\langle v \sigma^{2300}\right\rangle\left(Y_{2} Y_{3}-\bar{Y}_{2} \bar{Y}_{3}\right)+\left\langle v \sigma^{2311}\right\rangle\left(Y_{2} Y_{3}-Y_{1}^{2} \frac{\bar{Y}_{2} \bar{Y}_{3}}{\bar{Y}_{1}^{2}}\right) \\
& -\frac{1}{2}\left\langle v \sigma^{11 k 0}\right\rangle\left(Y_{1}^{2}-Y_{k} \frac{\bar{Y}_{1}^{2}}{\bar{Y}_{k}}\right)+\left\langle v \sigma^{k 110}\right\rangle\left(Y_{1} Y_{k}-Y_{1} \bar{Y}_{k}\right)+\Gamma_{k k^{\prime}}\left(Y_{k}-Y_{k^{\prime}} \bar{Y}_{k^{\prime}}\right)-\Gamma_{k^{\prime} k}\left(Y_{k^{\prime}}-Y_{k} \frac{\bar{Y}_{k^{\prime}}}{\bar{Y}_{k}}\right),
\end{aligned}
$$

where $k=2,3, k^{\prime}=5-k$, and

$$
\Gamma_{k k^{\prime}}=\sum_{\alpha \in k} g_{\alpha} m_{\alpha}^{2} \frac{K_{1}\left(m_{\alpha} / T\right)}{\left.\sum_{\beta \in k} g_{\beta} m_{\beta}^{2} K_{2}\left(m_{\beta} / T\right)\right)} \Gamma_{\alpha \rightarrow k^{\prime}, \mathrm{SM}}^{0},
$$

where $\Gamma_{\alpha \rightarrow k^{\prime}, \mathrm{SM}}^{0}$ is the partial decay width of the decay for one DM into another DM and SM particle, and $m_{\alpha}$ are the DM masses. $K_{j}(x)$ are the Bessel functions of the second kind and order $j$.

\section{B. Indirect detection}

The total number of events corresponding to DM pair annihilation is

$$
N=\sum_{i, j} \rho_{i}^{N} \rho_{j}^{N}\left\langle v \sigma^{i j}\right\rangle\left(1-\frac{1}{2} \delta_{i j}\right),
$$

where

$$
\begin{gathered}
\rho_{i}^{N}=\rho \xi_{i} / m_{i}, \\
\rho^{N}=\sum_{i} \rho_{i}^{N}
\end{gathered}
$$

are, respectively, the number densities for $\mathrm{DM}_{i}$ and the total DM number density. $\xi_{i}$ is the relative contribution of $\mathrm{DM}_{i}$ to the total DM density, $\xi_{i}=\Omega_{i} / \Omega_{\text {tot }}$, and $\xi_{1}+\xi_{2}=1$ for two-component models. $\left\langle v \sigma^{i j}\right\rangle$ is the short-hand notation for the cross section for annihilation of $\mathrm{DM}_{i} \mathrm{DM}_{j}$ into at least one SM final state. We can define an effective annihilation cross section,

$$
\langle v \sigma\rangle=\frac{2 N}{\rho^{N 2}} .
$$

To define the production rate of particles $a$ (here photons or antiprotons) from DM annihilation, we keep the same notation as for one-component DM,

$$
Q^{a}(E)=\frac{1}{2}\langle v \sigma\rangle \rho^{N^{2}} \frac{d N^{a}(E)}{d E},
$$

where the total yield reads

$$
Q^{a}(E)=\sum_{i \leq j}\left(1-\frac{1}{2} \delta_{i j}\right) \rho_{i}^{N} \rho_{j}^{N}\left\langle v \sigma^{i j}\right\rangle \frac{d N_{i j}^{a}(E)}{d E},
$$

and $d N_{i j}^{a} / d E$ is the energy distribution of particle $a$ produced in $\mathrm{DM}_{i} \mathrm{DM}_{j}$ annihilation, while $d N^{a} / d E$ is the effective spectrum for particle $a$.

The contribution of a given channel to the total cross section, for example, $\operatorname{Br}(A A W W)$ for $\tilde{A} \tilde{A} \rightarrow W W$, relates the annihilation cross section for that process to the total cross section,

$$
\operatorname{Br}(A A W W)=\frac{\left\langle v \sigma^{A A W W}\right\rangle \rho_{2}^{N^{2}}}{\langle v \sigma\rangle \rho^{N^{2}}} .
$$

\section{CONSTRAINTS}

\section{A. Theoretical constraints}

(i) Perturbativity

We impose the condition that the vertex factor in the Feynman rules for quartic interactions must be smaller than $4 \pi$. This ensures that the one-loop corrections are smaller than the tree-level contributions [124]. This imposes an upper bound on the quartic couplings; if a coupling enters several vertices, we choose the strongest bound. This leads to the condition, 


$$
\begin{aligned}
& \lambda_{2}<\frac{2 \pi}{3}, \quad\left|\lambda_{3}\right|<4 \pi, \quad\left|\lambda_{3}+\lambda_{4}\right|<4 \pi, \\
& \left|\lambda_{4} \pm \lambda_{5}\right|<8 \pi, \quad\left|\lambda_{3}+\lambda_{4} \pm \lambda_{5}\right|<4 \pi, \\
& \left|\lambda_{5}\right|<2 \pi, \quad\left|\lambda_{S 1}\right|<4 \pi, \quad\left|\lambda_{S 2}\right|<4 \pi, \\
& \left|\lambda_{S}\right|<\pi, \quad\left|\lambda_{S}^{\prime}\right|<\frac{\pi}{3} \text {. }
\end{aligned}
$$

(ii) Perturbative unitarity

For all scalar-scalar scattering processes at high energy, the partial wave unitarity condition should be satisfied for all scattering amplitudes. We impose the condition that the eigenvalues of the scattering matrices derived in [69] should all be smaller than $8 \pi$.

(iii) Stability conditions of the potential

To ensure that the quartic potential is bounded from below, we write the matrix of quartic interactions in a basis of non-negative field variables (in the $r^{2}, s^{2}$ basis defined in [69]) and demand this matrix to be copositive [125]. Thus, we obtain the following general stability conditions:

$$
\begin{gathered}
\lambda_{1}>0, \quad \lambda_{2}>0, \quad \lambda_{S}-\left|\lambda_{S}^{\prime}\right|>0, \\
\lambda_{3}+2 \sqrt{\lambda_{1} \lambda_{2}}>0, \\
\lambda_{3}+\lambda_{4}-\left|\lambda_{5}\right|+2 \sqrt{\lambda_{1} \lambda_{2}}>0, \\
\sqrt{\Lambda_{11} \Lambda_{22}}+\Lambda_{12}>0,
\end{gathered}
$$

where

$$
\begin{aligned}
\Lambda_{11}= & \lambda_{1} \cos ^{4} \gamma+\lambda_{2} \sin ^{4} \gamma \\
& +\left(\lambda_{3}+\left(\lambda_{4}+\lambda_{5} \cos (2 \Phi)\right) \rho^{2}\right) \cos ^{2} \gamma \sin ^{2} \gamma
\end{aligned}
$$

$$
\Lambda_{22}=\lambda_{S}+\lambda_{S}^{\prime} \cos \left(4 \Phi_{S}\right),
$$

$$
\begin{aligned}
\Lambda_{12}= & \frac{1}{2}\left[\lambda_{S 1} \cos ^{2} \gamma+\lambda_{S 2} \sin ^{2} \gamma\right. \\
& +\rho \sin \gamma \cos \gamma\left(\lambda_{S 21} \cos \left(\Phi-2 \Phi_{S}\right)\right. \\
& \left.\left.+\lambda_{S 12} \cos \left(\Phi+2 \Phi_{S}\right)\right)\right] .
\end{aligned}
$$

The last condition has to hold for all values of the arbitrary parameters $\rho, \gamma, \Phi, \Phi_{S}$ within the respective range $0 \leq|\rho| \leq 1,0 \leq \gamma \leq \frac{\pi}{2}, 0 \leq \Phi \leq 2 \pi$, and $0 \leq$ $\Phi_{S} \leq 2 \pi$ [69]. It is sufficient to compute the minimum of $\sqrt{\Lambda_{11} \Lambda_{22}}+\Lambda_{12}$ and check that it is positive.

(iv) Two stable dark matter candidates

To ensure two stable particles, it is necessary to prevent the decay channel $\tilde{A} \rightarrow \tilde{S} \tilde{S}$. Since this process is allowed for our choice of $Z_{4}$ charges, we impose the condition $M_{A}<2 M_{S}$.

(v) Electroweak precision tests (EWPT)

The electroweak precision parameters $\mathrm{S}, \mathrm{T}$ are sensitive to physics beyond the SM, and in this model, the one-loop contribution arises solely from the new scalar doublet and thus are similar to the IDM,

$$
\begin{aligned}
S= & \frac{1}{72 \pi} \frac{1}{\left(x_{2}^{2}-x_{1}^{2}\right)^{3}}\left[x_{2}^{6} f_{a}\left(x_{2}\right)-x_{1}^{6} f_{a}\left(x_{1}\right)\right. \\
& \left.+9 x_{2}^{2} x_{1}^{2}\left(x_{2}^{2} f_{b}\left(x_{2}\right)-x_{1}^{2} f_{b}\left(x_{1}\right)\right)\right],
\end{aligned}
$$

where

$$
\begin{aligned}
& f_{a}(x)=-5+12 \log (x), \\
& f_{b}(x)=3-4 \log (x),
\end{aligned}
$$

and $x_{1}=M_{H} / M_{H^{+}}, x_{2}=M_{A} / M_{H^{+}}$. When $x_{1}=x_{2}$, $S=\frac{1}{9 \pi} \log \left(x_{1}\right)$,

$$
\begin{aligned}
T= & \frac{1}{32 \pi^{2} \alpha v^{2}}\left[f_{c}\left(M_{H^{+}}^{2}, M_{A}^{2}\right)+f_{c}\left(M_{H^{+}}^{2}, M_{H}^{2}\right)\right. \\
& \left.-f_{c}\left(M_{H}^{2}, M_{A}^{2}\right)\right],
\end{aligned}
$$

where

$$
\begin{aligned}
& f_{c}(x, y)=\frac{x+y}{2}-\frac{x y}{x-y} \log \left(\frac{x}{y}\right), \quad x \neq y, \\
& f_{c}(x, y)=0, \quad x=y .
\end{aligned}
$$

Assuming a SM Higgs boson mass $m_{h}=125 \mathrm{GeV}$, the values of the $\mathrm{S}$ and $\mathrm{T}$ parameters in the limit $U=0$, have been determined to be

$$
S=0.06 \pm 0.09, \quad T=0.1 \pm 0.07,
$$

with a correlation coefficient +0.91 [126]. The EWPT data prefer a modest value for the mass splitting between the charged and neutral components of the doublet, roughly $\Delta^{+}<500 \mathrm{GeV}$ as can be seen in Fig. 4. When scanning over the parameter space, we have computed $\mathrm{S}$ and $\mathrm{T}$ for each point and have required that they fall within the ellipse corresponding to the $95 \%$ CL limit.

\section{B. Collider constraints: LEP}

In the low mass range, LEP experiments constrain the parameter space of the model; these limits are similar to the ones obtained in the IDM,

(i) Widths of $W$ and $Z$ bosons

The precise measurements of the $W$ and $Z$ widths impose that the decay of the gauge bosons, 

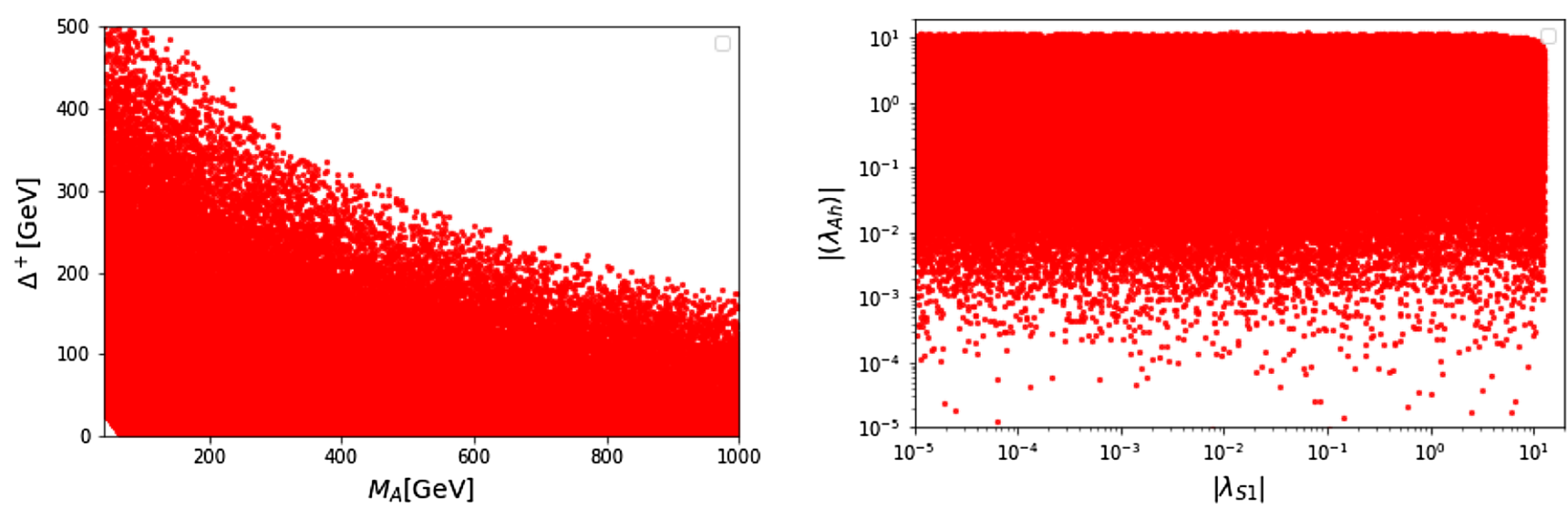

FIG. 4. Values of the mass difference $\Delta^{+}$and of the couplings of the two DM to the Higgs, $\lambda_{A h}, \lambda_{S 1}$, that satisfy all theoretical constraints together with LEP and electroweak precision constraints.

$W^{+} \rightarrow \tilde{H} \tilde{H}^{+}, \tilde{A} \tilde{H}^{+}$and $Z \rightarrow \tilde{A} \tilde{H}, \tilde{H}^{+} \tilde{H}^{-}$should be kinematically forbidden; thus,

$$
\begin{aligned}
M_{A}+M_{H^{+}}>M_{W}, & M_{H}+M_{H^{+}}>M_{W}, \\
M_{H}+M_{A}>M_{Z}, & 2 M_{H^{+}}>M_{Z} .
\end{aligned}
$$

(ii) Dijets and dileptons

The process $e^{+} e^{-} \rightarrow \tilde{H} \tilde{A}$ could lead to a visible dijet or dilepton signal from $\tilde{H} \rightarrow \tilde{A} \bar{f} f$; a reinterpretation of searches for neutralino pair production in Ref. [127] rules out the region defined by the intersection of the conditions below:

$$
\begin{gathered}
M_{H}<80 \mathrm{GeV}, \quad M_{A}<100 \mathrm{GeV}, \\
\text { if } M_{A}-M_{H}>8 \mathrm{GeV} .
\end{gathered}
$$

(iii) Charged Higgs

The process $e^{+} e^{-} \rightarrow \tilde{H}^{+} \tilde{H}^{-}$at LEP2 sets a limit $M_{H^{+}}>70 \mathrm{GeV}$ as a result of the reinterpretation of limits on charginos [128]. The lower limit on the charged Higgs is more stringent in the case of a compressed spectra. If the charged Higgs is long lived with a lifetime $\tau>10^{-6} \mathrm{sec}$, searches for heavy stable charged particles further restricts the charged Higgs to be $M_{H^{+}}>100 \mathrm{GeV}$ [129]. Moreover it also constrains lighter charged Higgses if their lifetimes are such that $95 \%$ of them have decayed before $3 \mathrm{~m}$. For $M_{H^{+}}=70 \mathrm{GeV}$, this corresponds to $\tau>3.1 \times 10^{-9}$ sec.

Applying theoretical constraints (stability and unitarity) as well as electroweak precision test limits and LEP constraints for the general scan leads to an upper limit on the mass difference within the scalar doublet, Fig. 4 (left), while the couplings of the two DM to the Higgs cover the whole range used in the scan, Fig. 4 (right).

\section{Collider constraints: LHC}

Precise measurements of the properties of the Higgs at the LHC further constrain the scalar sector, only two channels can be affected in the Z4IDSM.

(i) Invisible decay of the Higgs

For light DM, the SM Higgs can decay into two DM scalar particles. The direct 95\% CL limit on the invisible width was set by CMS at 19\% [130] using the vector boson fusion process. A more stringent limit was obtained by ATLAS by combining searches for an invisible Higgs produced in vector fusion or in association with top quarks at $13 \mathrm{TeV}$ with various searches at 7 and $8 \mathrm{TeV}$ [131]. We use the latter limit,

$$
\operatorname{Br}(h \rightarrow \text { invisible })<11 \% .
$$

(ii) The diphoton decay rate

The charged Higgs contributes to the one-loop induced process $h \rightarrow \gamma \gamma$ decay. The corresponding partial decay width is computed within micrOMEGAs [132], and this is compared with the experimental upper bound using HiggsSignals [133].

Finally, new physics searches at the LHC allow one to probe the model; those are monojet searches, disappearing track searches, searches for heavy stable charged particles (HSCP) and dileptons plus missing transverse energy (MET).

(i) ATLAS monojet search

The processes that lead to a monojet signature include $p p \rightarrow \tilde{A} \tilde{A} j$, as in the IDM model and $p p \rightarrow \tilde{S} \tilde{S}^{\dagger} j$. A recast of the monojet search was performed in $[134,96]$ in the IDM. The exclusion in the $\lambda_{A h}-M_{\mathrm{DM}}$ plane for different luminosities shows a sensitivity around $\lambda_{A h}=0.03$ for $M_{\mathrm{DM}}<$ $60 \mathrm{GeV}$ which degrades to $\mathcal{O}(1)$ as soon as the $\mathrm{SM}$ invisible Higgs threshold is crossed. We have used the exclusion for $20-100 \mathrm{fb}^{-1}$ as well as the projection for $3 a b^{-1}$. They have little impact on the 
parameter space of the Z4IDSM model. We have also estimated the contribution of the singlet scalar which for $M_{S}=M_{A}$ and $\lambda_{S 1}=\lambda_{A h}$ is twice that for the pseudoscalar doublet. As we see in the following, the higher mass and the different range of couplings allowed entail that the singlet does not contribute to present or future monojet searches at the LHC. An analysis of the monoZ and monoH processes in [96] also finds that only the region where $M_{H}<60 \mathrm{GeV}$ could be probed even with the high luminosity $\left(3 \mathrm{ab}^{-1}\right)$. Thus, we ignore these two processes in the following.

(ii) $j j+$ MET from vector boson fusion

The processes that lead to this signature include $p p \rightarrow \tilde{A} \tilde{A} j j$, as in the IDM model, and $p p \rightarrow$ $\tilde{S} \tilde{S}^{\dagger} j j$. In the inert doublet model, a recast of a search for an invisible Higgs produced through vector boson fusion (VBF) from the CMS Collaboration in Run 2 [130] was performed in Ref. [135]. The VBF processes was found to be more constraining than the above mentioned monojet search from a recast of the ATLAS Collaboration result [136] based on CHECKMATE [137,138]. The VBF process rules out only the region $\lambda_{H h}>1(3)$ for $M_{H} \approx 65(70) \mathrm{GeV}$ and only $\lambda_{H h}>10$ for $M_{H} \approx 85-100 \mathrm{GeV}$. We expect similar limits to be obtained on $\lambda_{A h}$ in the case where the pseudoscalar is the DM. In the following, we see that such large values of $\lambda_{A h}$ are not allowed by other constraints in the Z4IDSM model; thus, this process does not restrict further the parameter space of the model. We also checked that additional contributions involving the singlet scalar were always much suppressed relative to the doublet contribution, hence would not give additional constraints.

(iii) Long-lived particles

When the mass splitting between the charged Higgs and DM is small (at most few hundred MeVs), the charged Higgs is long lived. There are two collider searches aimed at long-lived particles that can be relevant in this model: the search for heavy charged particles (HSCP) and the disappearing track search which corresponds to the charged Higgs decaying into soft particles and DM with $c \tau \approx m$. To compute reliably the lifetime of the charged Higgs, we modify the model to include the effective vertices $\tilde{H}^{+} \tilde{A} \pi^{-}$ and $\tilde{H}^{+} \tilde{H} \pi^{-}$as described in Sec. II, including pions in the decay mode decreases significantly the lifetime of the charged Higgs. This effective vertex is taken into account only if the mass splitting lies in the range $140 \mathrm{MeV} \leq \Delta^{+} \leq 500 \mathrm{MeV}$. For larger mass splittings, a complete calculation would also include the decay into two pions, and we have however found that even without this mode the lifetime of the charged Higgs was short enough to be outside the range of applicability of the disappearing track, see

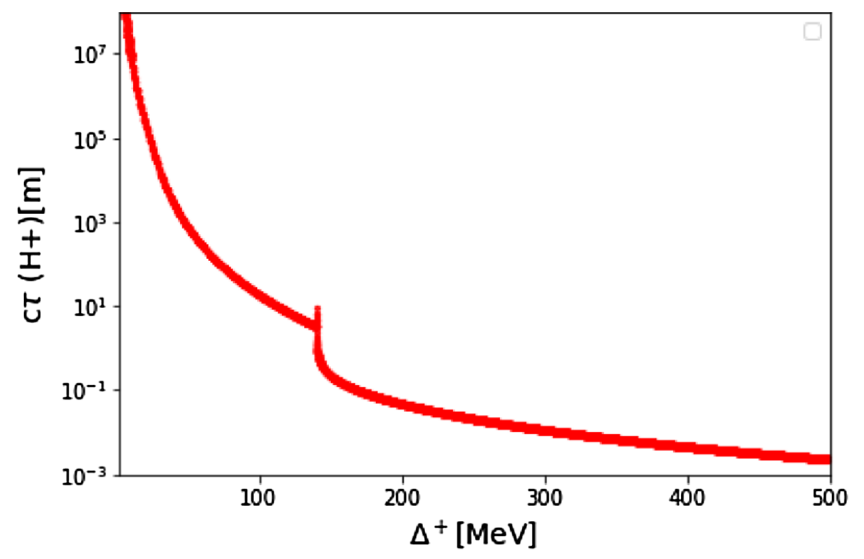

FIG. 5. $c \tau$ as a function of the mass splitting $\Delta^{+}=M_{H+}-M_{A}$.

Fig. 5. For our analysis, a precise knowledge of the charged Higgs lifetime is not required. We implemented the limits that were obtained in [115] for disappearing tracks. We also checked a posteriori that these limits were consistent with the ones included in the latest version of SModelS2.0.0 [139-141]. For very small mass splitting, we use the limits from HSCP searches based on $[142,143]$ that were implemented via SModelS2.0.0 [139,144].

(iv) Dilepton searches

Searches for opposite-sign dileptons and $E_{T}^{\text {miss }}$ at the LHC can constrain the IDM model. In this model, three processes contribute to dileptons, $p p \rightarrow$ $\tilde{A} \tilde{H}, \tilde{H}^{+} \tilde{H}^{-}, Z \tilde{H} \tilde{H}$, where dileptons come from the decay of the $\mathrm{Z}$ or from the decay $\tilde{H}^{+} \rightarrow \tilde{A} W^{+}$. The signature is similar to the one of neutralino/chargino in supersymmetry. A study of Run 1 [105] based on a recast of the SUSY search [145] showed that only the region where the lightest element of the doublet is lighter than $60 \mathrm{GeV}$ was constrained, the limit depending on the mass of other members of the doublet. In the Z4IDSM model, we expect similar results. Note that because these searches are targeted at supersymmetry the cuts applied may not be optimal for the IDM model; a study showed [146] that in the IDM the $E_{T}^{\text {miss }}$ peaked at much lower energy such that a softer cut on $E_{T}^{\text {miss }}$ has the potential to increase the sensitivity in the IDM model, thus to the Z4IDSM model. Moreover, analyses of the IDM at the high luminosity LHC have shown that multilepton channels could probe the model when the doublet DM is below the electroweak scale [147].

\section{Dark matter constraints}

\section{Relic density}

In this study, we require that both DM components explain DM, thus we require that the total relic density falls 
within the observed range determined very precisely by the PLANCK Collaboration to be $\Omega h^{2}=0.1184 \pm 0.0012$ [89]. We also take into account a $10 \%$ theoretical uncertainty; this rough estimate is motivated by previous studies performed in the inert doublet model, a model which shares many of the characteristics of the Z4IDSM, which showed that the full one-loop corrections to the relic density were typically of that order $[118,121]$. We take the $2 \sigma$ range; that is, we consider the following range for the total $\Omega_{\mathrm{DM}} h^{2}=\Omega_{1} h^{2}+\Omega_{2} h^{2}$;

$$
0.094<\Omega_{\mathrm{DM}} h^{2}<0.142 .
$$

The relic density and other DM observables are computed using micrOMEGAs5.3 [148,149].

\section{Direct detection}

Scalar DM interacts with nuclei only through spinindependent (SI) interactions. For DM masses near the electroweak scale, as considered here, the best limits on the SI cross section for DM scattering on nucleons have been obtained by XENON1T [1]. In the Z4IDSM, we compute the recoil energy distribution including the two DM signals scaled by their fractional contribution to the total DM density and use the recasted limits at 90\% CL implemented in micrOMEGAs [150]. For this, we use the default values for the astrophysical parameters, namely, a local DM density $\rho_{\text {DM }}=0.3 \mathrm{GeV} / \mathrm{cm}^{3}$ and a Maxwellian velocity distribution with the following parameters: the rotation velocity of the Galaxy, $v_{\text {Rot }}=220 \mathrm{~km} / \mathrm{s}$, the escape velocity of the Galaxy, $v_{\text {esc }}=544 \mathrm{~km} / \mathrm{s}$, the velocity of the Earth in the Galactic plane, $v_{\text {Earth }}=232 \mathrm{~km} / \mathrm{s}$.

\section{Indirect detection}

Fermi-LAT observations of photons from dwarf spheroidal galaxies provide one of the most robust constraints on DM especially at masses below the electroweak scale when DM annihilates into fermion pairs ( $b b$ or $\tau \tau)$. In our model, as is discussed later, the DM annihilation cross sections are, in general, largest for the gauge bosons final states. Thus, we compute $\langle v \sigma\rangle$ for $\tilde{A} \tilde{A}$ into $W W$ and $Z Z$ final states and require that $\langle v \sigma\rangle_{V V} \equiv\left\langle v \sigma^{A A V V}\right\rangle=$ $\left\langle v \sigma^{A A W W}\right\rangle+\left\langle v \sigma^{A A Z Z}\right\rangle$ lies below the 95\% CL given by FermiLAT in [2]. This limit ranges from $\langle v \sigma\rangle_{W W}>3 \times$ $10^{-26}-2.5 \times 10^{-25} \mathrm{~cm}^{3} / \mathrm{s}$ for DM masses ranging from 100-1000 GeV. Note that the photon spectrum from $Z Z$ final states is slightly below that of $W W$, so we ignore this difference. This approach is conservative as other channels can contribute as well as which is discussed in Sec. VA 2.

Searches for antiprotons performed by AMS-02 [151] provide the most severe constraints on WIMP DM. However, these constraints strongly depend on the cosmic ray $(\mathrm{CR})$ propagation parameters as well as on the DM profile. The uncertainty on the $\mathrm{CR}$ propagation parameters have been greatly reduced by AMS- 02 measurements of the boron to carbon ratio $(B / C)[152,153]$. Here again, we adopt a conservative approach and include only the gauge bosons final states. For this, we use the limits derived in [154] for $W W$ final states, and apply it to both the $W W$ and $Z Z$ final states, the antiproton spectrum from $W W$ and $Z Z$ being close to each other. In [154], the size of the diffusion halo is set to the minimum value $L=4.1 \mathrm{kpc}$, and the remaining propagation propagators and the exclusion cross section in the $W W$ channel for each DM mass are obtained from a global fit to $\mathrm{B} / \mathrm{C}$ and to the antiproton spectrum. The fit is done for three DM profiles, and we use the most conservative limit obtained assuming a generalized Navarro-Frenk-White (NFW) profile with $\rho=0.3 \mathrm{GeV} / \mathrm{cm}^{3}$ and also display the limit for a standard NFW profile with $\rho=0.38 \mathrm{GeV} / \mathrm{cm}^{3}$. The former gives an upper limit in the range $3 \times 10^{-26}-2 \times 10^{-25} \mathrm{~cm}^{3} / \mathrm{s}$, while the latter improves the limit in the range $9.4 \times 10^{-27}-6 \times 10^{-26} \mathrm{~cm}^{3} / \mathrm{s}$ for DM masses below $1 \mathrm{TeV}$.

Limits on DM annihilation will also be obtained by CTA which measures the photon spectrum albeit at higher energies [9]. We perform a dedicated analysis to determine the parameter space of the model that is within reach of CTA. For this, we use the combined photon spectra from all annihilation channels as described in Sec. III B.

\section{RESULTS}

To determine the parameter space of the model compatible with all current theoretical and experimental constraints, we have performed random scans over the masses and couplings in Eq. (3), first using a wide range for all parameters (the general scan) and then in the second case restricting to the region where the doublet is nearly degenerate in mass. Although this case requires to impose the conditions that some parameters are very small, the different DM and collider phenomenologies warrant a separate investigation. In both cases, the relic density and direct detection from XENON1T form stringent and robust constraints on the model, while current indirect detection constraints are marginal when taking a conservative approach. The LHC can probe the model mainly when the spectrum of the doublet is compressed. We also illustrate the potential of future DM searches to probe the model parameter space.

\section{A. General scan}

The free parameters of this model are varied randomly using a logarithmic scan for the couplings and a linear scan for the masses and/or mass splittings. We have already shown that the theoretical constraints, including EWPT, require that the mass differences among the components of the doublet cannot be too large, Fig. 4. Thus, we used as free variables the mass of the doublet DM, $M_{A}$, as well as the mass differences, $\Delta^{0}$ and $\Delta^{+}$. The range of the free 
TABLE I. Range of the free parameters of the Z4IDSM model used in the scan.

\begin{tabular}{lccccl}
\hline \hline$M_{A}$ & $40-1000 \mathrm{GeV}$ & $\left|\lambda_{S 1}\right|$ & $10^{-5}-4 \pi$ & $\lambda_{2}$ & $10^{-5}-2 \pi / 3$ \\
$\Delta^{0}$ & $0-500 \mathrm{GeV}$ & $\left|\lambda_{S 2}\right|$ & $10^{-5}-4 \pi$ & $\left|\lambda_{3}\right|$ & $10^{-5}-4 \pi$ \\
$\Delta^{+}$ & $0-500 \mathrm{GeV}$ & $\left|\lambda_{S 12}\right|$ & $10^{-5}-4 \pi$ & $\lambda_{S}$ & $10^{-5}-\pi$ \\
$M_{S}$ & $40-1500 \mathrm{GeV}$ & $\left|\lambda_{S 21}\right|$ & $10^{-5}-4 \pi$ & $\left|\lambda_{S}^{\prime}\right|$ & $10^{-5}-\pi / 3$ \\
\hline \hline
\end{tabular}

parameters are given in Table I. Note that $\lambda_{2}, \lambda_{S}, \lambda_{S}^{\prime}$ have no impact on the DM phenomenology. When presenting the results of the numerical scan, we first determine the impact of the relic density and direct detection on the parameter space of the model, in particular, on the mass range of the two dark matter and on their couplings. These results are to be contrasted with what is expected in the two submodels: the inert doublet and the complex singlet scalar mentioned in Sec. III. After having shown the impact of the current indirect detection searches, we estimate the potential of future indirect searches. We show that generally the annihilation into vector bosons is the dominant one, just as in the doublet model, although in some cases unusual DM annihilation modes are possible. We propose two benchmarks to illustrate these. Finally, we display the complementarity of future DD and ID searches in probing the model. Note that the role of the LHC is marginal and is mostly confined to the region where the spectrum is compressed that is analyzed separately in Sec. V B. Although we do not include the region where $M_{A}>2 M_{S}$ in our discussion since it leads to only a single DM, $\tilde{S}$, we have checked that as expected the single DM has the same properties as the one found in the complex singlet model, with the only allowed region for $M_{S} \approx m_{h} / 2$ or above the $\mathrm{TeV}$ scale.

\section{Relic density and direct detection}

The relic density constraint from PLANCK and the direct detection limits from XENON1T provide the most important and robust constraints on the model. Several

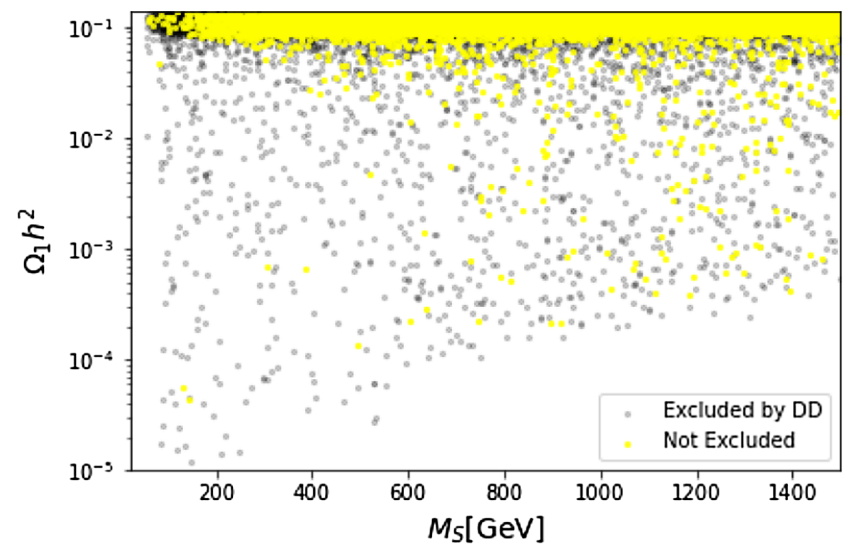

parameters and couplings can play roles in determining the relic density of each DM component as was discussed in Sec. III A. However, DM scattering on nuclei proceeds through Higgs exchange and is thus basically determined by the couplings to the Higgs, $\lambda_{S 1}$ for $\tilde{S}$ and $\lambda_{A h}$ for $\tilde{A}$. We find that generally the singlet forms the dominant DM component. Moreover it satisfies DD constraints for the full mass range above $M_{S}>58.3 \mathrm{GeV}$ in stark contrast with the singlet DM model. The doublet can be dominant only in a narrow region around $m_{h} / 2$ and for masses $M_{A}>500 \mathrm{GeV}$, see Fig. 6. These two regions correspond to the ones where in the IDM the DM relic density is compatible with the PLANCK measurement. The doublet component usually contributes to at least a few per-mil of the total relic density. It can however be suppressed in a few cases, and notably, we found a region for $M_{A}<250 \mathrm{GeV}$ where $\Omega_{2} h^{2} \approx 10^{-6}$. These correspond to configurations specific to the Z4IDSM model where important DM conversion or semiannihilation reduces both $\Omega_{1} h^{2}$ and $\Omega_{2} h^{2}$ when $M_{A}>M_{S}$. Values of $\Omega h^{2}<10^{-4}$ are also found in fine-tuned cases where the doublet or the singlet lies near the Higgs resonance and requires a very weak coupling to the Higgs that allows it to escape DD constraints.

To implement the DD constraint from XENON-1T, we recall that we have combined the signal from both DM. We first remark that even if $\tilde{A}$ is subdominant, it is still effectively probed by XENON1T, see Fig. 7 where we show the predictions for the spin independent cross section for each DM candidate over the allowed parameter space. Each contribution is rescaled by the appropriate fraction of the DM candidate, $\xi_{i}$. On the same figure, we also make a naive projection of the reach of XENON-nT and DARWIN where for simplicity we consider each DM signal separately. The vast majority of points are within the reach of XENON-nT for the doublet DM; however, in some cases, the signal is suppressed by two or three orders of magnitude. Points in red that lie below the line showing the XENON-nT projection in Fig. 7 left (right) are within reach

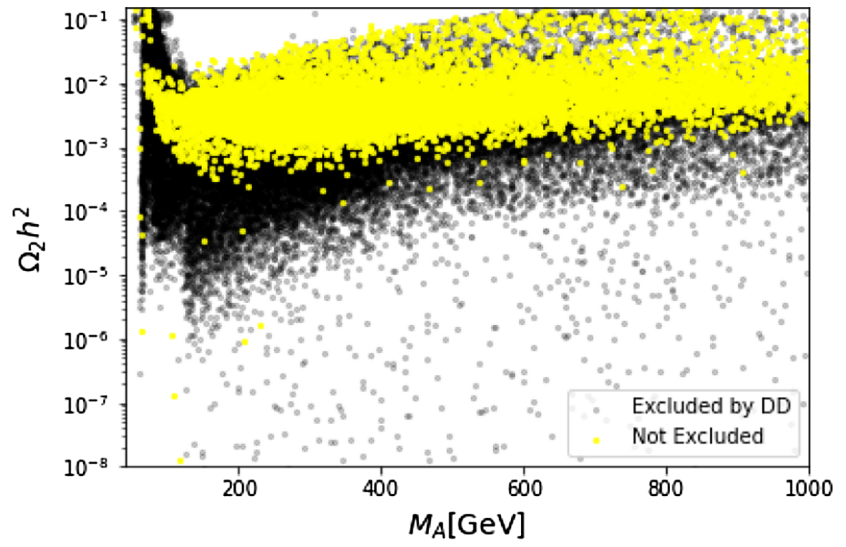

FIG. 6. $\Omega_{1} h^{2}$ (left) and $\Omega_{2} h^{2}$ (right) as a function of the corresponding DM mass, for all points satisfying theoretical, collider, and the total relic density constraints (gray) as well as DD constraint from XENON-1T (yellow). 



FIG. 7. Spin-independent DM proton scattering cross section times the fraction of DM component, as a function of the corresponding DM masses, $\sigma_{S p} \xi_{1}-M_{S}$ (left) and $\sigma_{A p} \xi_{2}-M_{A}$ (right). The black (red) line shows the projected reach of XENON-nT (DARWIN). Points in yellow (red) are within the reach of XENON-nT (DARWIN), and blue points are beyond the reach of DARWIN.
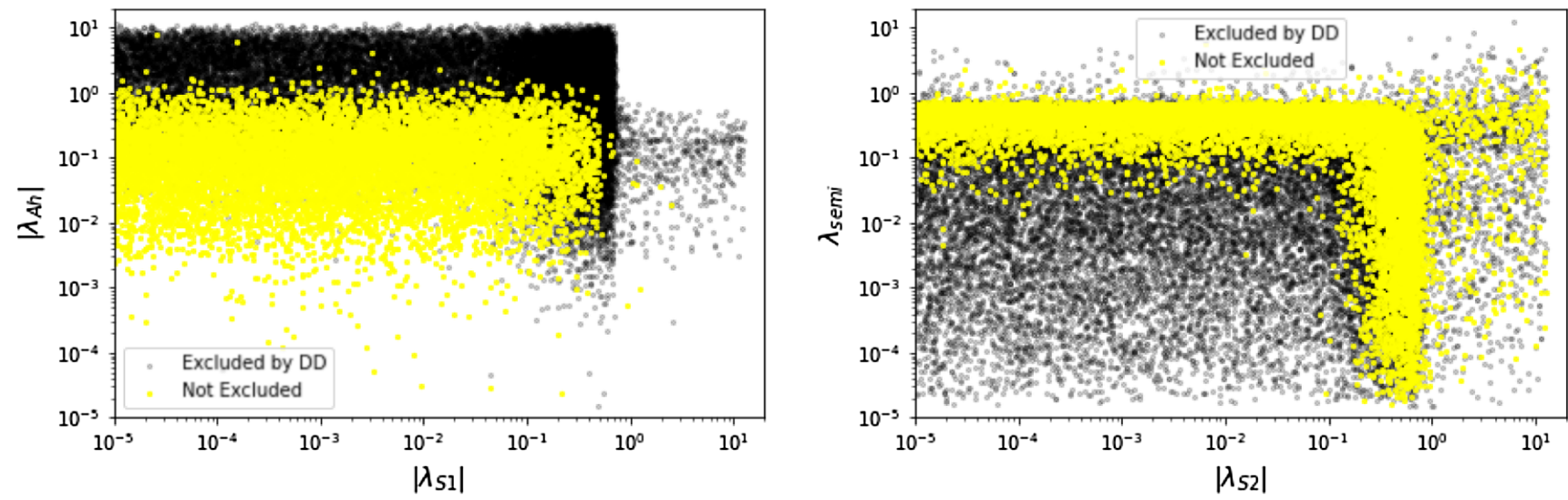

FIG. 8. Allowed points in the $\lambda_{A h}-\lambda_{S 1}$ plane (left) and $\lambda_{\text {semi }}-\lambda_{S 2}$ plane (right). Points in black are excluded by XENON-1T.

of XENON-nT for the doublet (singlet) component. Clearly, the singlet component often easily escapes DD even if it forms the dominant DM. This is due to the presence of the conversion/semiannihilation channels which allow one to reduce the value of $\lambda_{S 1}$ as compared to the case where only interactions with the SM set the relic density of the singlet. Indeed, $\lambda_{S 1}$ can be as small as $10^{-5}$, the lower limit in our scan, see Fig. 8 (left panel). We find that some amount of semiannihilation or DM conversion is necessary to satisfy both relic density and direct detection since the region where both $\lambda_{\text {semi }}$ and $\left|\lambda_{S 2}\right|$ are small is ruled out by DD constraint, see Fig. 8 (right panel). Indeed if semiannihilation and DM conversion are negligible, the large value of $\lambda_{S 1}$ required by the relic density constraint is excluded by DD, unless $M_{S}$ is above the TeV scale. Note that the product of couplings $\lambda_{A h} \times \lambda_{S 1}$ can also lead to DM conversion through processes of the type $\tilde{S} \tilde{S}^{\dagger} \rightarrow \tilde{A} \tilde{A}$; however, we found that these couplings are constrained by DD to be $\lambda_{A h} \times \lambda_{S 1}<5 \times 10^{-2}$. Thus, in general, DM conversion is dominated by $\lambda_{S 2}$. Similarly, the coupling $\lambda_{A h}$ responsible for DM formation for the doublet component covers a wider range than in the IDM, and the largest values are excluded by DD as seen in Fig. 8 (left). ${ }^{2}$ After applying all current constraints, we also note that the bulk of the points are in the region where $0.1<\lambda_{S 2}<1$ with extension all the way to the upper limit. Similarly, $0.1<\lambda_{\text {semi }}<1$, and these conditions are required to suppress the abundance of the singlet without having a too large $\lambda_{S 1}$. In Fig. 8 (left), we clearly see that large values of $\lambda_{S 1}$ are disfavored, although $\lambda_{S 1}$ can reach 1 when it is associated with a rather heavy DM, thus evading DD constraints.

\section{Indirect detection: Photons and antiprotons}

The contributions of the final states for which the cross section can reach at least $10^{-27} \mathrm{~cm}^{3} / \mathrm{s}$ are shown in the Appendix A. These include pair annihilation of $\tilde{A}$ into

\footnotetext{
${ }^{2}$ Note that since we performed a random scan with a linear dependence on the mass differences between the singlet and the doublet components, we have a sparse sampling of a very compressed spectrum; hence, we have a sparse sampling of the small values of $\lambda_{A h}$. This case is covered in Sec. V B.
} 


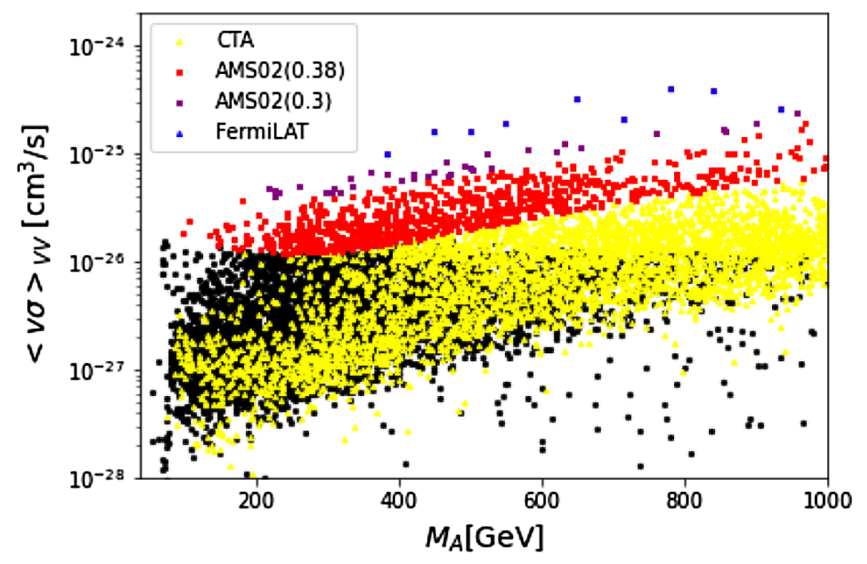

FIG. 9. Points excluded by FermiLAT (blue) and AMSO2 assuming NFW and $\rho=0.3$ (purple) or $\rho=0.38$ (red) in the $\langle v \sigma\rangle_{V V}-M_{A}$ plane. The reach of CTA is also shown (yellow). Points in black are beyond the reach of these detectors.

$W(Z)$ pairs as well as semiannihilation channels such as $\tilde{S} \tilde{S} \rightarrow \tilde{H}^{-} W^{+}$or $\tilde{H}^{+} W^{-}$or processes involving interactions between the two dark sectors such as $\tilde{S} \tilde{S} \rightarrow \tilde{H}^{+} \tilde{H}^{-}$. We found that the cross sections that are in the realm of FermiLAT are to a large extent only possible in the gauge bosons channel; thus, to impose these constraints, we include only the VV channels where we sum over the $W$ and $Z$ final states. We find that FermiLAT excludes the region with the largest cross sections for large $M_{A}$ as displayed in Fig. 9.

To impose the constraint from AMS02, we follow the analysis in [154] which gives the excluded cross section from the WW channel assuming different profiles. We assume the NFW profile and a cored generalized NFW profile [154], the latter leading to the most conservative limit. The scenarios that are currently exclude by AMSO2 under these assumptions are also displayed in Fig. 9. Clearly, the uncertainty on the limits coming from the assumption on the profile is large. This further justifies our approximation of similar spectra from $Z Z$ and $W W$ channels and of neglecting the semiannihilation channels. Note that values of $M_{A}$ up to $1 \mathrm{TeV}$ (the upper limit in our scan) can be probed by AMS02 for the largest cross sections.

Next, we determine whether the increased sensitivity of future detectors might allow one to probe scenarios where $\langle v \sigma\rangle$ is dominated by semi annihilation and or DM conversion processes. First, we illustrate the impact of nonSM channels on the photon spectrum using the two benchmarks in Table II, see Fig. 10. These are chosen as examples of scenarios where the dominant contributions to $\langle v \sigma\rangle$ come from non-SM final states. For the first benchmark, the semiannihilation couplings are of $\mathcal{O}(1)$; hence, semiannihilation forms the dominant channels, with $\tilde{S}^{\dagger} \tilde{A} \rightarrow$ $Z \tilde{S}$ (44\%), $\tilde{S} \tilde{S} \rightarrow W^{-} \tilde{H}^{+}$(20\%), $\tilde{S} \tilde{S} \rightarrow W^{+} \tilde{H}^{-}$(12\%), $\tilde{S} \tilde{S} \rightarrow Z \tilde{A}(18 \%)$, and $\tilde{S}^{\dagger} \tilde{A} \rightarrow h \tilde{S}(6 \%)$. The total cross section $\langle v \sigma\rangle=7 \times 10^{-26} \mathrm{~cm}^{3} / \mathrm{s}$, and for all energies except for $E_{\gamma}>200 \mathrm{GeV}$, the full spectrum is dominated by $\tilde{S}^{\dagger} \tilde{A} \rightarrow Z \tilde{S}$. Moreover, we find that the photon spectrum for channels with the $\tilde{H}^{ \pm} W^{\mp}$ final state is very soft. For this benchmark, the spectrum is such that $\tilde{H}^{ \pm} W^{\mp}$ cannot be produced on shell; moreover, the mass splitting between the charged and neutral Higgs do not allow for the charged Higgs to decay into a real $W$. The decay rather proceeds via the 3-body final state; thus, the photon spectrum is soft. For the second benchmark, the spectrum is slightly heavier, and the mass difference $\Delta^{+}$is only a few GeVs. As just argued, the photon spectrum associated with the dominant channel $\tilde{S} \tilde{S} \rightarrow W^{-} \tilde{H}^{+}(46 \%)$ is very soft. With only a $18 \%$ contribution from $\tilde{A} \tilde{A} \rightarrow Z Z$ to the total annihilation, it is the channel that dominates the photon spectrum. The channels $\tilde{S}^{\dagger} \tilde{A} \rightarrow h \tilde{S}(20 \%)$ and $\tilde{S}^{\dagger} \tilde{A} \rightarrow Z \tilde{S}$ (6\%) give subdominant contributions. Note that both of these benchmarks are within the reach of CTA.

We include all channels in the analysis that determines the reach of CTA. For this, we follow the analysis in [155]. For an arbitrary spectrum, a table containing the contribution to the total likelihood from each energy bin is provided. We compute the total spectrum with micrOMEGAs for each point in our scan and compare with the total annihilation cross section that can be probed for a given DM mass. Doing so, we find that CTA will significantly extend the reach of FermiLAT and AMS in probing the model. This is illustrated in Fig. 9 which shows the exclusion in the $\langle v \sigma\rangle_{V V}-M_{A}$ plane. Note that all points that are currently excluded by AMS-02 for the two hypothesis we considered for the DM density are also within the reach of CTA. Note that all the points that are within reach of CTA have a total $\langle v \sigma\rangle>3 \times 10^{-26} \mathrm{~cm}^{3} / \mathrm{s}$, and those with the low values for $\langle v \sigma\rangle_{V V}$ in Fig. 9 are in fact dominated by one of the semiannihilation channels. Moreover, in some cases, some large values of $\langle v \sigma\rangle$ remain beyond reach. This occurs either because invisible channels give a large contribution or because dominant channels lead to soft photons as illustrated for the $\tilde{H}^{+} W^{-}$final state in the benchmarks above. Note also that CTA is not sensitive to thermal cross sections for DM masses below

TABLE II. Benchmark points.

\begin{tabular}{|c|c|c|c|c|c|c|c|c|c|}
\hline & $M_{S}$ & $M_{A}$ & $M_{H}$ & $M_{H^{+}}$ & $\lambda_{S 1}$ & $\lambda_{S 2}$ & $\lambda_{3}$ & $\lambda_{S 12}$ & $\lambda_{S 21}$ \\
\hline $\mathrm{A}$ & 244.3 & 411.9 & 449.8 & 418.5 & $3.68 \times 10^{-4}$ & 0.470 & $9.07 \times 10^{-5}$ & 1.86 & 0.98 \\
\hline $\mathrm{B}$ & 328.1 & 544.4 & 758.4 & 551.1 & $-1.29 \times 10^{-2}$ & $-1.02 \times 10^{-3}$ & $-3.18 \times 10^{-2}$ & -0.0103 & -1.16 \\
\hline
\end{tabular}



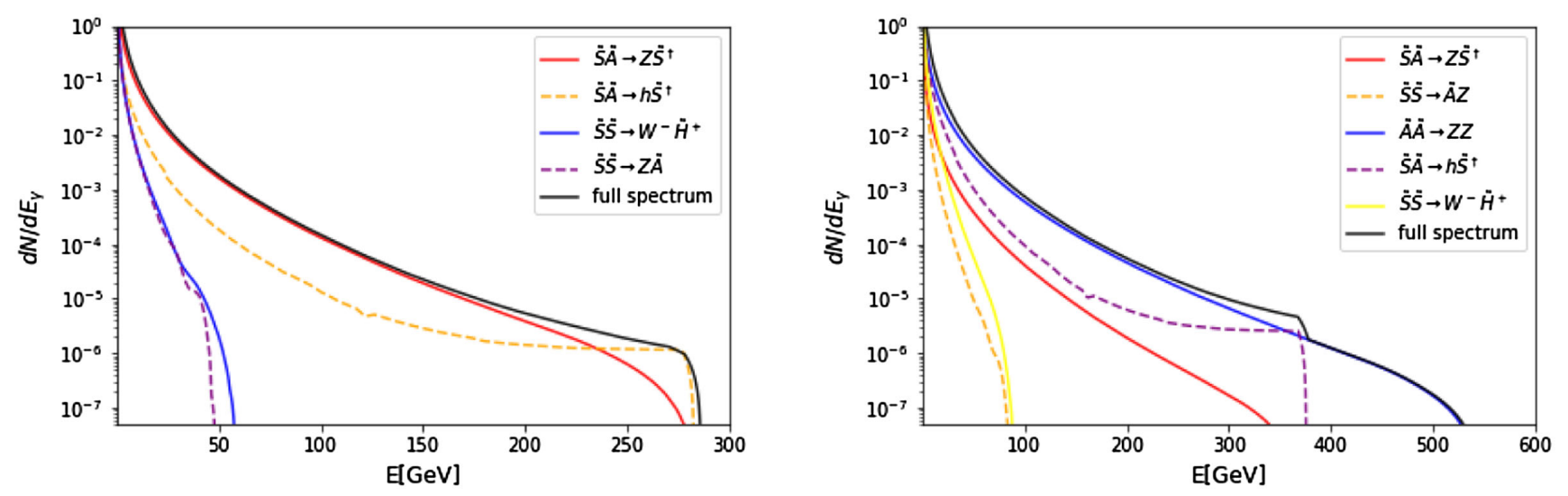

FIG. 10. Photon spectrum for the two benchmarks A and B of Table II.

about $200 \mathrm{GeV}$ when it annihilates into $W$ pairs [155]; hence, many points below $200 \mathrm{GeV}$ cannot be probed.

\section{Colliders and direct and indirect detection}

Before doing a comparison of the reach in future direct and indirect searches, we briefly discuss collider constraints. For this, we consider only the points that are not excluded by other constraints (theoretical, relic density, and Xenon-1T). To evaluate the current and future probes of the model through monojet, we use the analysis of [134] performed in the IDM. We computed separately the cross section for monojet production corresponding to $p p \rightarrow$ $\tilde{A} \tilde{A} j$ as well as $p p \rightarrow \tilde{S} \tilde{S} j$. The former is driven mainly by $\lambda_{A h}$, while the latter depends on $\lambda_{S 1}$. Since $\sigma(p p \rightarrow$ $\tilde{A} \tilde{A} j)=1 / 2 \sigma(p p \rightarrow \tilde{S} \tilde{S} j)$ for $\lambda_{A h}=\lambda_{S 1}$ and $M_{A}=M_{S}$, we simply translated the limit on $\lambda_{A h}$ in a limit on $\lambda_{S 1}$. These limits together with our parameters points are displayed in Fig. 11 in the planes $\lambda_{A h}-M_{A}$ and $\lambda_{S 1}-M_{A}$, respectively. We find that the values of $\lambda_{A h}$ and/or $\lambda_{S 1}$ that survive theoretical and DM constraints are always below the expected reach of the LHC. In particular, note that the singlet contribution to the monojet is always far below

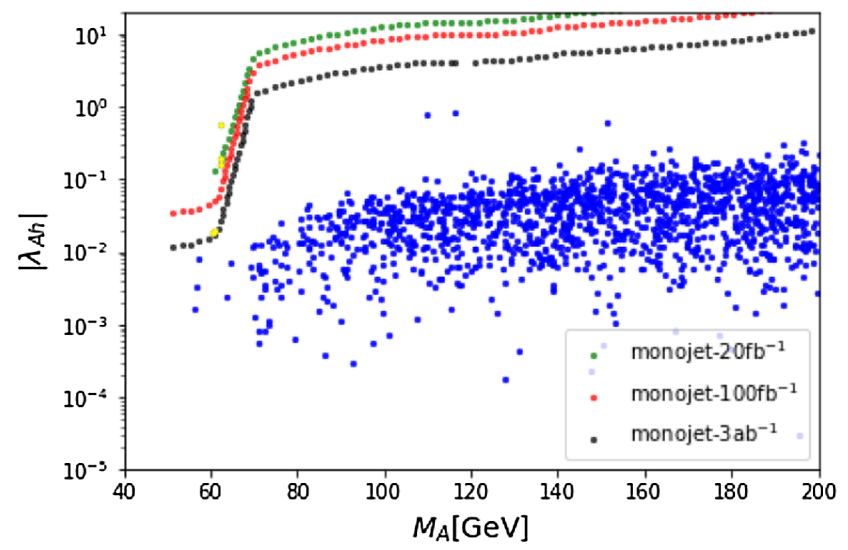

the experimental limit especially since we find few points in the low mass region. The approximation of computing separately the doublet and singlet contributions is therefore valid. Thus, we conclude that the monojet search cannot probe the Z4IDSM once other theoretical or dark matter constraints are taken into account.

Finally, we perform a comparison of the direct and indirect detection reaches that we have discussed separately in Secs. VA 2 and VA 1. Figure 12 shows the reach for XENON-nT, CTA, and DARWIN in the $\langle v \sigma\rangle_{V V}-M_{A}$ plane for points that pass all constraints including those from FermiLAT and AMS02. The same points are plotted in the $\sigma_{A p} \xi_{2}-M_{A}$ plane in Fig. 12 (right). A large fraction of the points are within reach of both CTA and XENON-nT and/or DARWIN. This large overlap between the DD and ID probes opens the possibility of cross checking a DM signal; nevertheless, the parameter space will not be fully covered. XENON-nT will probe basically all scenarios where $\lambda_{A h} \geq 0.1$ or $\lambda_{S 1}>0.071$, while DARWIN will improve these by roughly a factor 2 . CTA will extend the reach to cases where the SI signal for both DM is weak, notably for the doublet near the TeV scale. CTA however does not cover completely the region with lighter masses of the doublet

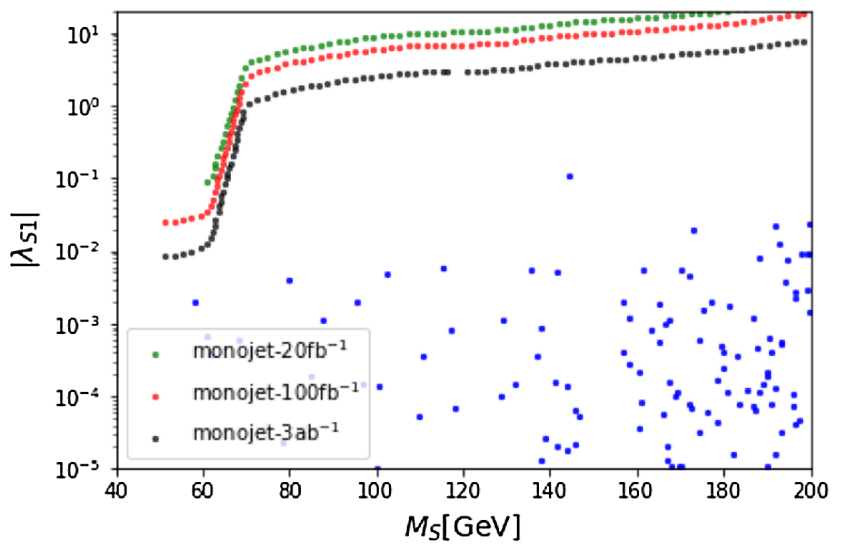

FIG. 11. Monojet limit at LHC for $\mathcal{L}=20,100,3000 \mathrm{fb}^{-1}[134]$ and allowed points for the Z4IDSM model (blue) in the $\left|\lambda_{A h}\right|-M_{A}$ and $\left|\lambda_{S 1}\right|-M_{S}$ planes. 

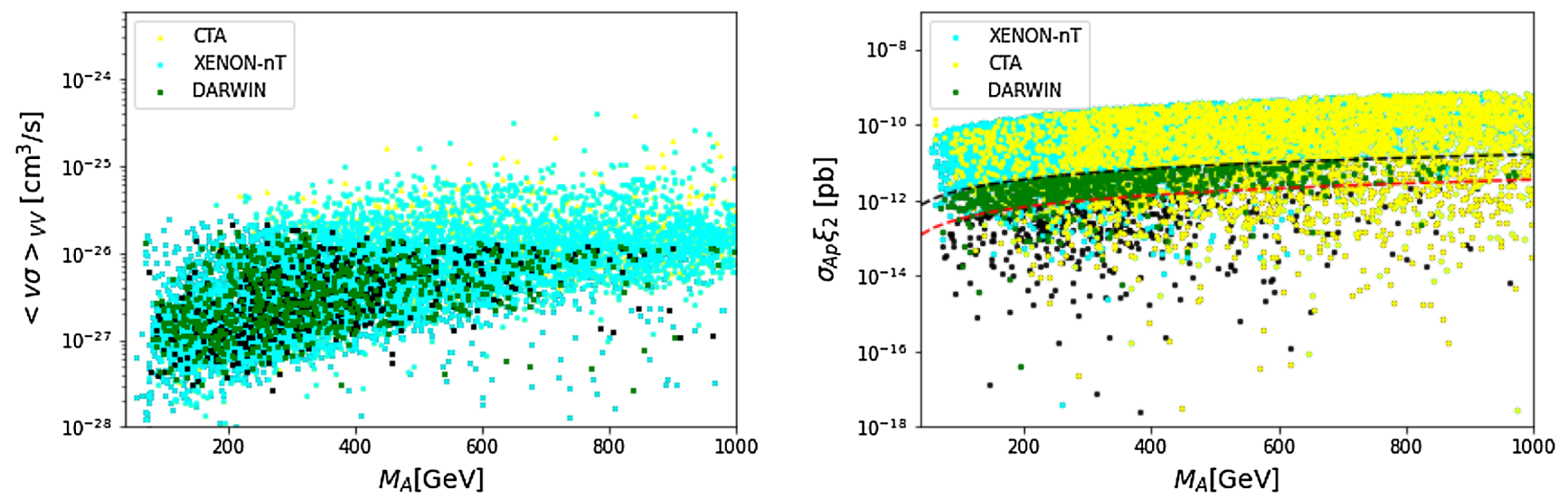

FIG. 12. Future reach of direct detection with XENON-nT (cyan) and DARWIN (green) and indirect detection with photons (CTA) in the $\langle v \sigma\rangle_{V V}-M_{A}$ (left) and $\sigma_{A p} \xi_{2}-M_{A}$ (right) planes. Points in black are beyond the reach of these detectors. In the right panel, the projected exclusions for XENON-nT (black dashed line) and DARWIN (red dashed line) in the case of one component DM are also shown.

(below $300 \mathrm{GeV}$ ), both because the signal is suppressed by the small value of $\xi_{2}$ and because the sensitivity is not as good. In fact, there are cases that cannot be probed, even if both $M_{S}$ and $M_{A}$ are below $300 \mathrm{GeV}$. This is typically because the couplings $\lambda_{A h}$ and $\lambda_{S 1}$ are small. For these points either semiannihilation or DM conversion plays an important role. We found scenarios that are out of reach of future detectors for any of the full the DM masses considered; in general, they feature a subdominant doublet DM with $\Omega_{2} h^{2}$ between .001 and .02 .

To conclude this section, we found that the interactions between the dark sectors play important roles in allowing a singlet DM of any mass. We also found that current DD and ID results mainly constrained the subdominant doublet component and that current LHC monojet limits had no impact. In the next section, we show with a dedicated analysis of the compressed doublet that other LHC signatures can effectively probe the parameter space of the model.

\section{B. The nearly degenerate doublet}

In order to investigate more closely the case where the doublet is nearly degenerate, we performed an independent scan. A compressed spectrum opens up the possibility of three DM candidates; indeed, for very small mass splittings between the two neutral component of the doublet, both can be stable and contribute to the relic density. To investigate this possibility more precisely, we assume that the two neutral components of the doublets are nearly degenerate, and we treat the mass splitting of the charged component as a free parameter in the range

$$
\Delta^{+}=1-500 \mathrm{MeV} \text {. }
$$

Note that we set $\Delta^{0}=201 \mathrm{keV}$ corresponding to the minimum value that prevents inelastic scattering on nucleons, a process which is strongly constrained since it depends on the gauge coupling $\tilde{A} \tilde{H} Z$ [82]. In this case, $\tilde{H}$ will be nearly stable and can be treated as a third DM component when computing the relic density.

The remaining masses are varied randomly in the ranges $40 \mathrm{GeV}<M_{S}<1.5 \mathrm{TeV}$ and $70 \mathrm{GeV}<M_{A}<1 \mathrm{TeV}$, while we use the range defined in Table I for the couplings. Note that with this choice of mass splitting among the neutral components of the doublet, the coupling $\lambda_{5} \approx$ $10^{-7}-10^{-6}$. Thus the coupling of the scalar and the pseudoscalar to the Higgs are nearly equal $\lambda_{A h} \approx \lambda_{H h}$. This entails that the contributions of $\tilde{H}$ and $\tilde{A}$ to the relic density are nearly equal.

\section{Collider constraints}

As mentioned in Sec. IV C, the new charged scalar can be long lived and lead to a signature in disappearing track or HSCP. Current LHC limits exclude long-lived $\tilde{H}^{+}$with masses below $550 \mathrm{GeV}$, see Fig. 13. The mass limit weakens for a shorter lifetime; for example, when $c \tau \approx 10 \mathrm{~m}$ the mass limit drops to $500 \mathrm{GeV}$ and when $c \tau \approx 0.5 \mathrm{~m}$ only charged Higgs lighter than $200 \mathrm{GeV}$ are excluded. The disappearing track search targets lower lifetimes $c \tau<0.3 \mathrm{~m}$ and can reach only up to $M_{H^{+}} \approx 250 \mathrm{GeV}$. Note that the disappearing track search at LHC does not cover the region $M_{H^{+}}<100 \mathrm{GeV}$. The typical mass split that is constrained by HSCP is below $150 \mathrm{MeV}$, while the disappearing track can apply for larger $\Delta^{+}$up to $280 \mathrm{MeV}$, see Fig. 13 (right).

Note that, as we discuss in the next section, constraints from DM observables lead to a more stringent limit on the coupling $\lambda_{A h}$ as compared to the general case described in Sec. VA 3. Thus, we do not expect significant constraints from monojet searches, see also [134].

\section{DM observables: Relic density and direct detection}

After imposing collider constraints, we explore DM observables. To solve for the DM relic density, we use 

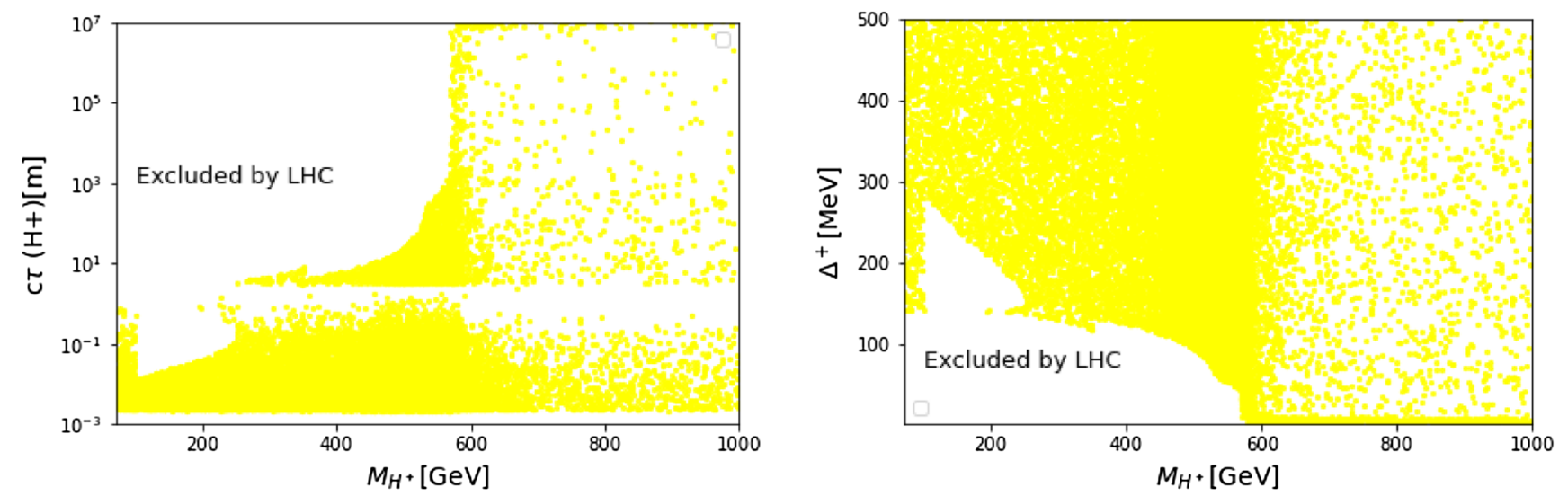

FIG. 13. LHC exclusion from disappearing tracks and HSCP in the $c \tau-M_{H^{+}}$plane (left) and $\Delta^{+}-M_{H^{+}}$plane (right) after applying relic density and direct detection constraints from XENON1T; allowed points are in yellow.

Eq. 13 that include a third DM component, $\tilde{H}$. Because we have fixed $\Delta^{0}$ to be very small, $\tilde{H}$ has not decayed at the time of DM formation. We compute its relic density and find that it is approximately the same as that of $\tilde{A}$. In the following results, we understand $\Omega_{2}=\Omega_{H}+\Omega_{A}$ to be the total contribution of the doublet to the relic density. Note however that this third component will decay into $\tilde{A}$ and will not be present in the Universe today. To check this, we have solved the abundance equations, Eq. 13, until $T=10^{-13} \mathrm{GeV}$ and found $Y_{\tilde{H}}$ to be negligible.

As known from the IDM, the relic density of the compressed doublet can be in agreement with PLANCK for masses above roughly $500-600 \mathrm{GeV}$. This is the main feature that we recover in the Z4IDSM compressed model, Fig. 14 (right). For lighter masses, the doublet is a subdominant DM component, and $\Omega_{2}$ increases with $m_{A}$ up to about $500-600 \mathrm{GeV}$. This means that it is much easier to have a dominant doublet DM than in the general scan. For heavier masses of the doublet, we would expect the doublet to be overabundant. However, the effect of DM conversion and/or semiannihilation can help reduce

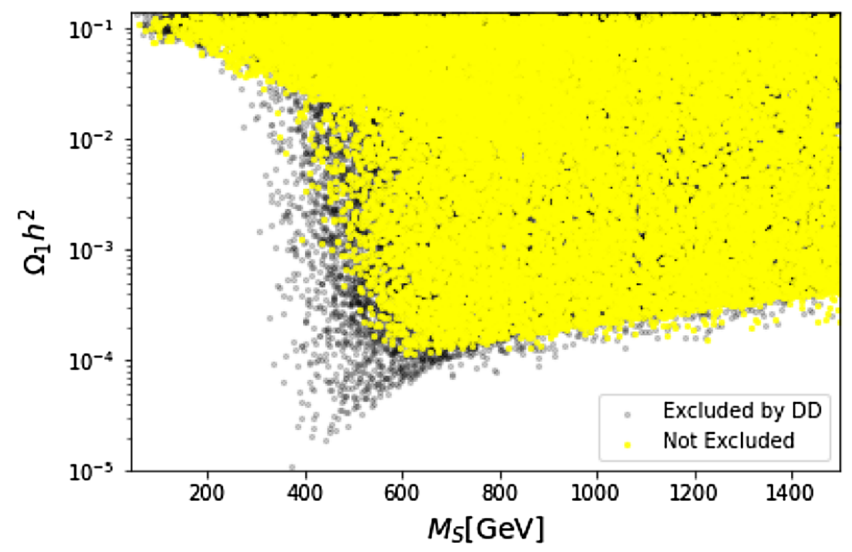

its relic density; thus, we find allowed scenarios for $M_{A}$ covering the full range of masses probed. At the same time, $\Omega_{1}$ is likely to be subdominant especially for masses above $500 \mathrm{GeV}$.

The current constraints and future reach of DD experiments are displayed in Fig. 15. We see, in particular, that a much larger fraction of points can be probed by XENONnT through the singlet component as compared with the general scan. Those are the points in yellow that lie below the XENON-nT projected limit on $\sigma_{A p} \xi_{2}$ in Fig. 15 (right). This is tied to the main difference with the general scan which is found in the range of allowed values for the couplings $\lambda_{S 1}$ and $\lambda_{A h}$, see Fig. 16. In particular, with a compressed spectrum, it is possible to have $\lambda_{S 1}>1$, while escaping DD constraints. This occurs when $\tilde{S}$ forms a subdominant DM component, that is, $\Omega_{1} h^{2} \ll 0.1$, and its DD signal which is proportional to the DM fraction $\xi_{1}$ can be suppressed despite a large value of $\lambda_{S 1}$. This is only possible for $M_{S}>500 \mathrm{GeV}$ since it requires the doublet to be the dominant DM component, see Fig. 16 (left). When the doublet contributes significantly to the total DM

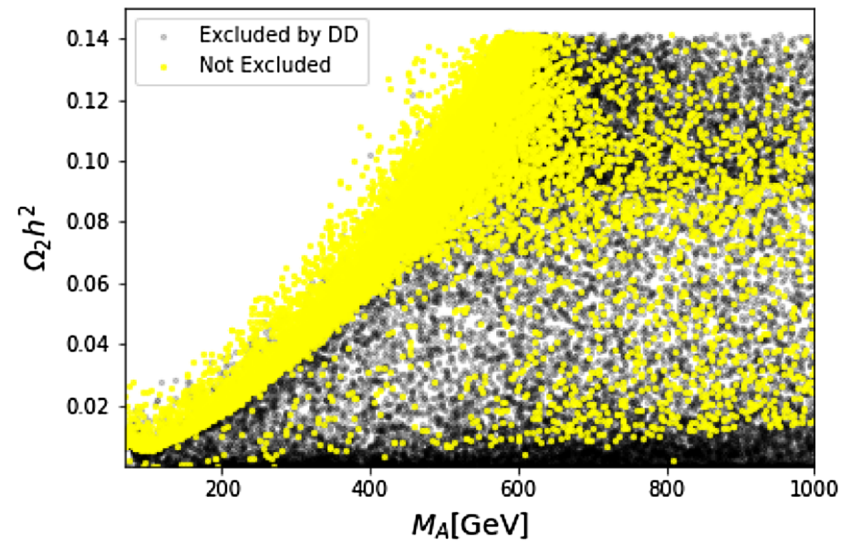

FIG. 14. $\Omega_{1} h^{2}$ (left) and $\Omega_{2} h^{2}$ (right) as a function of the corresponding DM mass, for all points satisfying theoretical, collider, and the total relic density constraints (gray) as well as DD constraint (yellow). 

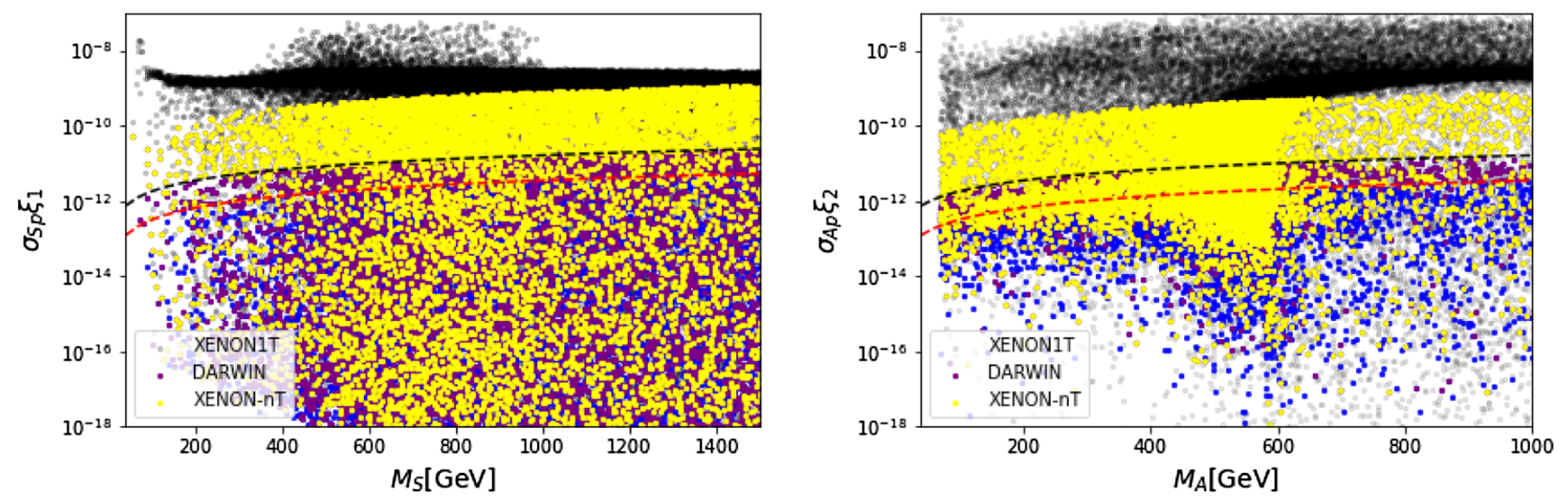

FIG. 15. Spin-independent DM proton scattering cross section normalized with the fraction of DM component, as a function of the corresponding DM masses, $\sigma_{S p} \xi_{1}-M_{S}$ (left) and $\sigma_{A p} \xi_{2}-M_{A}$ (right). The black (red) line shows the projected reach of XENON-nT (DARWIN). Points in yellow (purple) are within the reach of XENON-nT (DARWIN), and blue points are beyond the reach of DARWIN.
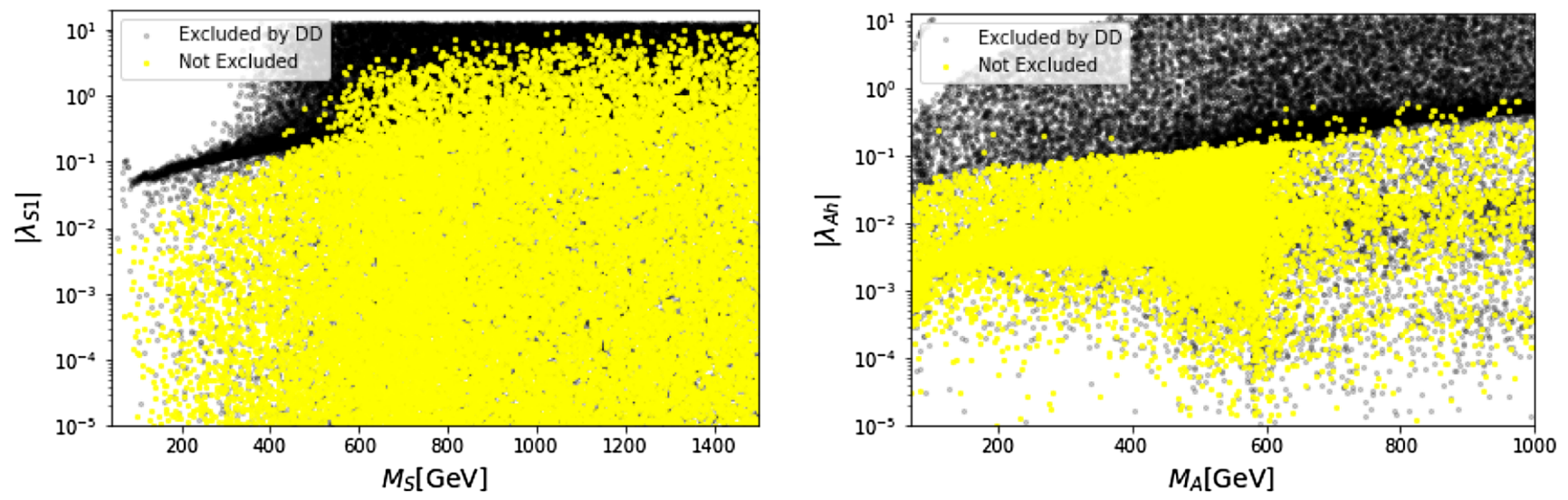

FIG. 16. All points satisfying theoretical, collider, and the total relic density constraints (gray) as well as DD constraint (yellow) in the $\lambda_{S 1}-M_{S}$ plane (left) and $\lambda_{A h}-M_{A}$ plane (right).

component, it cannot evade so easily DD constraints. Thus, the upper limit on the coupling $\lambda_{A h}<0.2$ that is set by the XENON-1T constraint is more stringent than for the general scan. Note that DD constraints are particularly strong when $M_{A}$ is in the range $400-600 \mathrm{GeV}$, and the doublet is the dominant DM component, as seen by the high density of points in Fig. 16 (right) in that region. As opposed to the general scan, it is not possible to have both $\tilde{S}$ and $\tilde{A}$ very heavy, Fig. 17 (left). In this region, despite the contributions of semiannihilation and DM conversion, large values for $\lambda_{S 1}$ and $\lambda_{A h}$ are required to ensure that both $\Omega_{i}$ are small enough. Such couplings are constrained by XENON1T.

With the compressed spectrum, we also find that some amount of semiannnihilation or DM conversion is necessary to evade DD constraints. Indeed DD imposes a lower bound on $\lambda_{\text {semi }}, \lambda_{S 2} \approx 0.1$ as can be seen in Fig. 17 (right). Moreover, we find that a much larger fraction of the allowed points are associated with $\lambda_{S 2}>1$ as compared to the general case. The reason for this was discussed in Sec. III; the assisted freeze-out mechanism in the presence of a large cross section $\sigma^{1122}$ will reduce the abundance of both DM components but more significantly that of the heavier component, which is generally the singlet. Therefore, for large $\lambda_{S 2}$, the abundance of the singlet will be very small; thus, the doublet has to account for all of the DM. This is hard to realize when the spectrum is not compressed since typically $\Omega_{2} h^{2} \ll 0.1$ even without the additional contribution from the DM conversion term. When the singlet is the lighter component, we also find allowed points with $\lambda_{S 2}>1$, and those also feature a large semiannihilation coupling $\lambda_{\text {semi }}>1$. In this case, processes such as $\tilde{S} \tilde{S} \rightarrow \tilde{A} Z, \tilde{A} h$ contribute significantly to bring $\Omega_{1} h^{2}$ below the PLANCK limit, while DM conversion processes suppress $\Omega_{2} h^{2}$.

In summary, with a compressed doublet spectrum, either $\tilde{S}$ or $\tilde{A}$ can be the dominant DM component, and both can be within reach of future DD detectors. However a large 



FIG. 17. Allowed points (yellow) in the $\left|\lambda_{A h}\right|-\left|\lambda_{S 1}\right|$ plane (left) and in the $\lambda_{\text {semi }}-\left|\lambda_{S 2}\right|$ plane (right). Also shown are points that are excluded by XENON-1T (black).

fraction of the model parameters remains beyond the reach of XENON-nT and even DARWIN. In part, this is related to the fact that, as mentioned above, the coupling of the doublet DM to the Higgs is suppressed, with much lower values of $\lambda_{A h}$ favored.

\section{Indirect detection}

The contribution of different channels to $\langle v \sigma\rangle$ in different channels are shown in Appendix B. The largest annihilation cross sections are again found for $\tilde{A} \tilde{A} \rightarrow W W / Z Z$; thus, to be conservative, we include only these channels to derive the FermiLAT and AMSO2 constraints. However, for detectors with increased sensitivity, such as CTA, it becomes more important to exploit all channels as is illustrated in the general scan. Recall that in the WW channel CTA is expected to probe $\langle v \sigma\rangle \approx 2(1) \times$ $10^{-26} \mathrm{~cm}^{3} / \mathrm{s}$ for $M_{\mathrm{DM}}=0.2(1) \mathrm{TeV}$. The constraints are displayed in terms of $\langle v \sigma\rangle_{V V}$ in Fig. 18 (left). FermiLAT only constrains a few scenarios corresponding to the largest annihilation cross section into WW, while AMS02 provides stronger constraints for $M_{A}$ up to $700 \mathrm{GeV}$ depending on the assumption on the DM profile and the local density. CTA will also probe the scenarios with large cross sections in WW and will extend the reach to the highest mass used in the scan $(1 \mathrm{TeV})$. Moreover because of the important contribution from semiannihilation channels, CTA will also probe many scenarios where the $W W / Z Z$ channels are suppressed. In Fig. 18 (right), one can see that the total $\langle v \sigma\rangle$ is always above $3 \times 10^{-26} \mathrm{~cm}^{3} / \mathrm{s}$ except when $M_{A} \approx$ $M_{h} / 2$, yet several scenarios remain out of reach of CTA, in particular, when $M_{A}<200 \mathrm{GeV}$. This is explained by the limited sensitivity of the experiment in that region and by the fact that the contribution from the $W W / Z Z$ channels which give generally the main signature can be quite suppressed. In addition, for a large number of points, DM conversion processes $\tilde{S} \tilde{S}^{\dagger} \leftrightarrow \tilde{A} \tilde{A}$ dominate DM annihilation, leaving no signature for indirect detection. This can occur for any value of $M_{A}$.
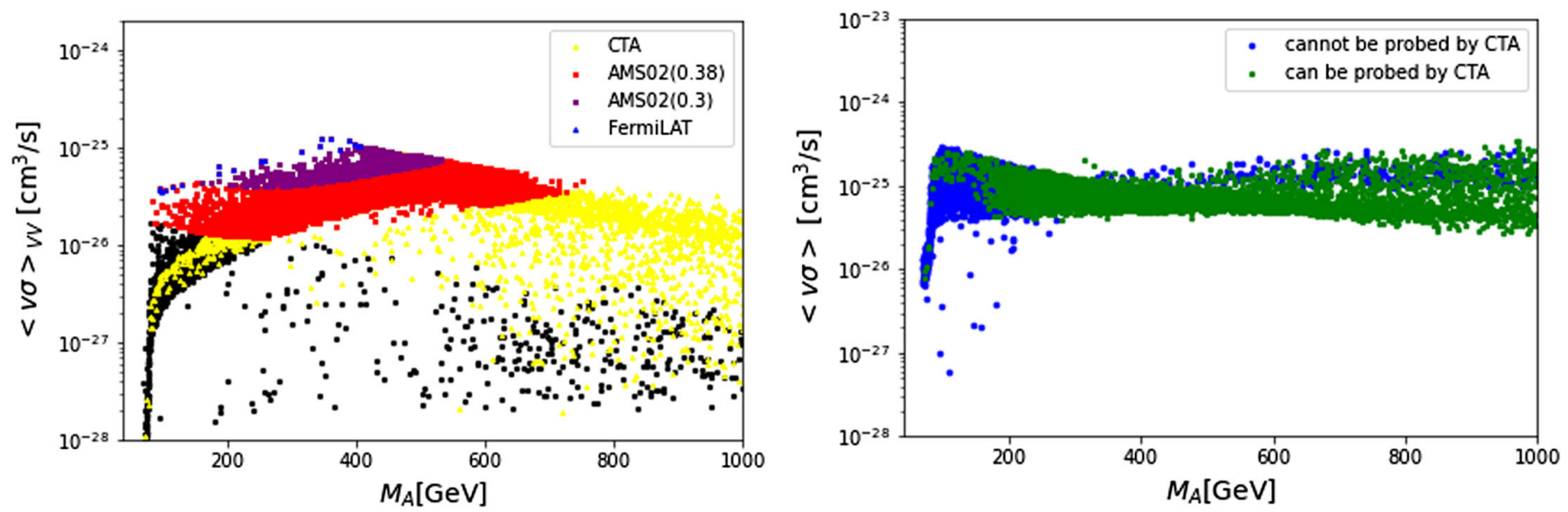

FIG. 18. Left: constraints on $\langle v \sigma\rangle_{V V}$ from FermiLAT (blue) and AMS02 assuming an NFW profile with $\rho_{\text {DM }}=0.3$ (purple) or a generalized NFW profile with $\rho_{\text {DM }}=0.38$ (red). The reach of CTA is also displayed (yellow). Black points are beyond the reach of these three indirect detection searches. Right: total $\langle v \sigma\rangle$ corresponding to the points that are within (green) or beyond (blue) the reach of CTA. 



FIG. 19. Reach of XENON-nT (blue), DARWIN (green), and CTA (yellow) for $\langle v \sigma\rangle_{V V}$ (left) and $\sigma_{\tilde{A} p} \xi_{2}$ (right) vs $M_{A}$. Only points that are allowed by current constraints are included, and for DARWIN, only points that are beyond the reach of XENON-nT are considered. Black points are beyond the reach of all detectors.

\section{Complementarity colliders, direct, and indirect detection}

To highlight the complementarity between the indirect and direct detection searches, we compare the future reaches of CTA, XENON-nT, and DARWIN in Fig. 19 expressed in terms of the quantities relevant for ID, $\langle v \sigma\rangle_{V V}$, or DD, $\sigma_{A p} \xi_{2}$. Most of the parameter space will be probed by future detectors. Clearly, the low mass region $\left(M_{A}<200 \mathrm{GeV}\right)$ will be better probed by DD detectors, since in this region CTA has a reduced sensitivity. From Fig. 19, it might seem that there is a large overlap for the points that are within reach of CTA and those that are within reach of XENON-nT or DARWIN; however, the two types of searches probe different scenarios. DD can probe scenarios with large values of $\lambda_{S 1}$ or $\lambda_{A h}$ as seen in Fig. 16; on the other hand, CTA can probe points where $\tilde{A} \tilde{A} \rightarrow W W$ is large or points with large values for $\lambda_{\text {semi }}$ and/or $\lambda_{S 2}$ by taking advantage of semiannihilation channels. The former requires that the doublet component, $\xi_{2}$, is not too suppressed, while the latter can depend on both $\xi_{1}$ and $\xi_{2}$ according to the dominant process.

A few benchmarks that illustrate this complementarity between ID and DD are shown in Table III for DM masses of a few hundred GeVs. All points have $\langle v \sigma\rangle>4 \times 10^{-26} \mathrm{~cm}^{3} / \mathrm{s}$; however, only $\mathrm{BP} 1$ and $\mathrm{BP} 3$ are within reach of CTA, due to the large contribution from $\tilde{A} \tilde{A} \rightarrow W W$ with $\xi_{2} \approx 0.5$ in both cases. BP1 is an example of a point with a large $\lambda_{S 2}$ and large contribution

TABLE III. Benchmarks for the compressed doublet.

\begin{tabular}{lcccc}
\hline \hline & $\mathrm{BP} 1$ & $\mathrm{BP} 2$ & $\mathrm{BP} 3$ & $\mathrm{BP} 4$ \\
\hline$M_{A}$ & 313.594 & 327.952 & 360.31 & 202.74 \\
$M_{H^{+}}$ & 313.727 & 328.265 & 360.81 & 203.01 \\
$M_{S}$ & 313.1 & 317.98 & 848.9 & 287.8 \\
$\lambda_{2}$ & 0.185 & $1.7 \times 10^{-2}$ & $7.5 \times 10^{-3}$ & $7.8 \times 10^{-2}$ \\
$\lambda_{3}$ & $1.03 \times 10^{-5}$ & $4.05 \times 10^{-2}$ & $2.5 \times 10^{-2}$ & $2.0 \times 10^{-4}$ \\
$\lambda_{S 1}$ & $2.98 \times 10^{-3}$ & $1.77 \times 10^{-4}$ & $1.32 \times 10^{-2}$ & $2.15 \times 10^{-3}$ \\
$\lambda_{S 2}$ & 3.94 & 4.01 & $6.17 \times 10^{-1}$ & $1.75 \times 10^{-3}$ \\
$\lambda_{S 12}$ & $1.5 \times 10^{-2}$ & $3.11 \times 10^{-3}$ & $1.75 \times 10^{-4}$ & $1.46 \times 10^{-1}$ \\
$\lambda_{S 21}$ & $4.08 \times 10^{-2}$ & $2.38 \times 10^{-1}$ & $7.3 \times 10^{-3}$ & $3.5 \times 10^{-3}$ \\
$\Omega_{1} h^{2}$ & 0.0641 & 0.112 & 0.0491 & 0.105 \\
$\Omega_{2} h^{2}$ & 0.0427 & 0.0146 & 0.0592 & 0.0114 \\
$\sigma_{\tilde{S} p}^{S I}(\mathrm{pb})$ & $8.0 \times 10^{-13}$ & $2.74 \times 10^{-15}$ & $2.2 \times 10^{-12}$ & $3.9 \times 10^{-13}$ \\
$\sigma_{\tilde{A} p}^{S I}(\mathrm{pb})$ & $6.8 \times 10^{-13}$ & $9.36 \times 10^{-11}$ & $1.2 \times 10^{-11}$ & $3.9 \times 10^{-12}$ \\
$\mathrm{DARWIN}$ & $\boldsymbol{x}$ & $\checkmark$ & $\checkmark$ & $\times$ \\
$\xi_{2}^{2}\langle v \sigma\rangle_{V V}\left(\mathrm{~cm}^{3} / \mathrm{s}\right)$ & $2.95 \times 10^{-26}$ & $2.37 \times 10^{-27}$ & $4.27 \times 10^{-26}$ & $1.67 \times 10^{-27}$ \\
$\langle v \sigma\rangle\left(\mathrm{cm}^{3} / \mathrm{s}\right)$ & $2.0 \times 10^{-25}$ & $9.39 \times 10^{-26}$ & $8.25 \times 10^{-26}$ & $1.17 \times 10^{-25}$ \\
$\mathrm{CTA}$ & $\checkmark$ & $\times$ & $\checkmark$ & $\times$ \\
$c \tau(\mathrm{m})$ & 4.2 & $9.9 \times 10^{-3}$ & $2.3 \times 10^{-3}$ & $7.8 \times 10^{-3}$ \\
\hline \hline
\end{tabular}



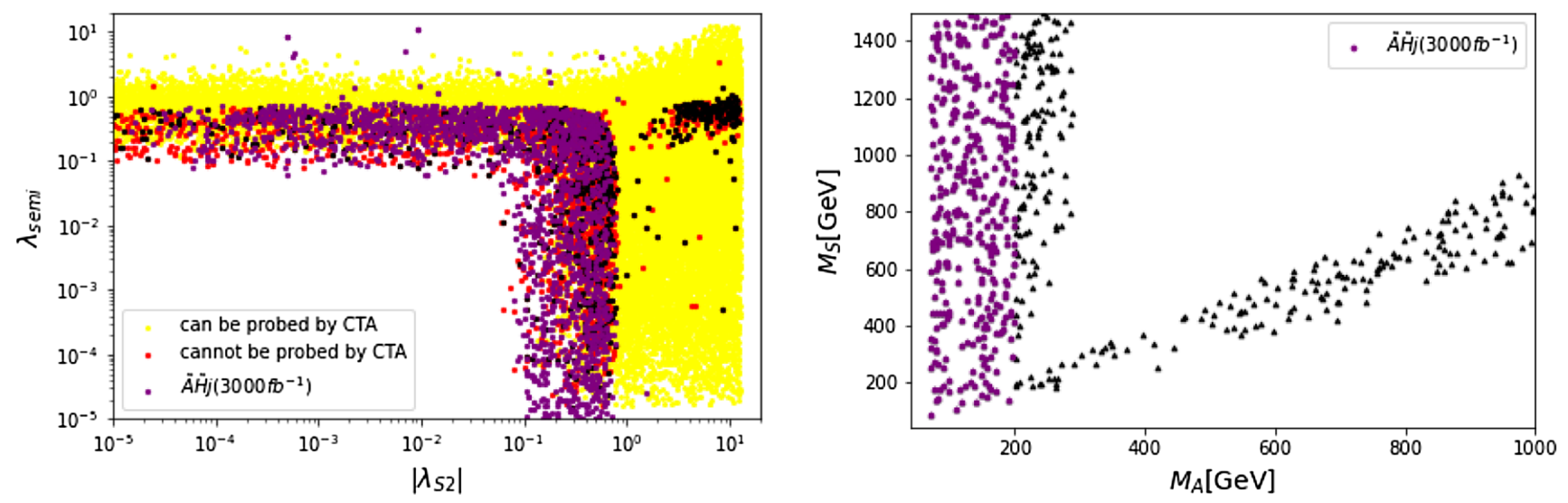

FIG. 20. Left: points that can (yellow) or cannot (red) be probed by CTA; in purple, are scenarios that are within reach of HL-LHC and black points that are beyond the reach of CTA,DARWIN, and HL-LHC. Right: points that are beyond the reach of CTA and DARWIN in the $M_{S}-M_{A}$ plane; in purple, are scenarios that are within the reach of HL-LHC.

from $\tilde{A} \tilde{A} \rightarrow \tilde{S} \tilde{S}^{\dagger}$ that has nevertheless a large enough contribution from $\tilde{A} \tilde{A} \rightarrow W^{+} W^{-}$. This point escapes future DD searches due to the small values of $\lambda_{3} \approx \lambda_{A h}$ and $\lambda_{S 1}$. $\mathrm{BP} 2$, on the other hand, with similar masses and values of $\lambda_{S 2}$, escapes CTA's reach due to the suppressed contribution of the doublet to dark matter. Moreover, since $\lambda_{3} \approx \lambda_{A h} \approx$ $10^{-2}$, the doublet component can be probed by DARWIN. BP3 features a similar value of $\lambda_{A h}$ and also has $\lambda_{S 1}$ large enough such that both the singlet and doublet components are within DARWIN's reach. Finally, BP4 is an example of a point that falls beyond the sensitivity of future detectors. Here, $\lambda_{3}$ and $\lambda_{S 1}$ are small, thus leading to weak DD signals, while semiannihilation processes driven by $\lambda_{S 12}$ ensure that the relic density constraint is satisfied. This point escapes CTA due to the somewhat suppressed $\langle v \sigma\rangle_{V V}$ and the fact that the mass of the doublet $200 \mathrm{GeV}$ falls in the region where the sensitivity of CTA is not maximal.

The scenarios that remain out of reach of the future detectors considered are concentrated in two regions: $M_{A}<$ $300 \mathrm{GeV}$ and $M_{A} / 2<M_{S}<M_{A}$, see Fig. 20 (right). For these scenarios, the singlet is the dominant DM component, as was the case for BP4. Because of the direct detection constraint, these scenarios have small values for both $\lambda_{S 1}$ and $\lambda_{A h}$, which are restricted to be below $0.01-0.03$, with the stricter bound for lighter $M_{A}$. Thus, an important contribution from semiannihilation and DM conversion is needed to satisfy the relic density constraint. Indeed, we find that a significant fraction of the points that are out of reach correspond to $\lambda_{S 2}>1$, and they fall in the region where $M_{S}<M_{A}$ and are invisible because of the large contribution from $\tilde{A} \tilde{A} \rightarrow \tilde{S} \tilde{S}^{\dagger}$. In the region $M_{A} \approx 200 \mathrm{GeV},\langle v \sigma\rangle$ can exceed $10^{-26} \mathrm{~cm}^{3} / \mathrm{s}$; however, the small value of $\Omega_{2}$ leads to a weaker signal that is not always compensated by a large contribution from semiannihilation channels. For example, for BP4, $\tilde{S} \tilde{S} \rightarrow V \tilde{D}$, where $\tilde{D}$ stands for any component of the doublet that has a cross section $\langle v \sigma\rangle=7.6 \times 10^{-27} \mathrm{~cm}^{3} / \mathrm{s}$.
Concerning the potential of the high luminosity LHC (HL-LHC) to probe the nearly degenerate scenario, we mention that an analysis of the $p p \rightarrow \tilde{A} \tilde{H} j$ leading to a monojet signature showed that the HL-LHC with a luminosity of $3 \mathrm{ab}^{-1}$ could constrain doublet DM masses up to $200 \mathrm{GeV}$ independently of the value of $\lambda_{A h}$ [134]. Such a region could also be probed at a future $100 \mathrm{TeV}$ collider [111]. This coverage is complementary to the one from astrophysical searches. In particular, it will allow one to cover the low mass region in Fig. 19 that will be mainly probed by a detector like DARWIN. Moreover, the HL-LHC would cover most of the unprobed region where the singlet is the heavier DM, see Fig. 20. Finally, note that searches for disappearing tracks at upcoming runs at LHC could allow one to probe some of the points that escape astrophysical and collider searches. For example, BP4 has $c \tau=0.78 \mathrm{~cm}$ and is just beyond the reach of current searches. Note however that $c \tau$ depends critically on the mass splitting which we chose here to be below $500 \mathrm{MeV}$.

\section{DISCUSSION}

Combining the dark matter sectors of the IDM and singlet model and allowing interactions between the dark sectors opens up significantly the possibility for DM compatible with stringent relic density and direct detection constraints. Because of the presence of seminnihilation and/or DM conversion processes, the relic density constraint can be satisfied with reduced couplings of each DM component to the Higgs, thus escaping the direct detection constraints. We have investigated the parameter space of the model that satisfies all current constraints and found that generally the singlet is the dominant DM component. We also found that the mass range up to the $\mathrm{TeV}$ scale can be satisfied for both DM components, while strong collider constraints preclude DM much below the electroweak scale. In fact, after taking into account direct detection and relic density constraints, the invisible width of the 
Higgs is sufficiently suppressed that it does not constrain the model further. We highlighted the complementarity of direct and indirect searches and stressed the importance of semiannihilation processes such as $\tilde{S}^{\dagger} \tilde{A} \rightarrow Z \tilde{S}, h \tilde{S}$ that can contribute significantly to the photon spectrum in indirect detection. After taking into account cosmological and astrophysical constraints and requiring that both DM components explain totally the relic density, we found that the conventional DM signatures at colliders, such as monojet, did not help in further probing the model. While future astrophysics searches will provide powerful probes of the model, some scenarios can still escape future searches by CTA and DARWIN. Typically, those scenarios have a doublet below $400 \mathrm{GeV}$ and feature a subdominant doublet component that has a suppressed coupling to the Higgs.

Since a compressed doublet can lead to a quite different phenomenology, we have investigated separately the case of a very degenerate doublet spectrum, while allowing the singlet to be any mass. In this case, the doublet can be the dominant dark matter component. In fact, for the very compressed spectrum that we considered, the two neutral components of the doublet contribute equally to the DM abundance at freeze-out temperature. For this, we solved the generalized Boltzmann equation for three DMs, although we present the results summing over the contributions of the two doublet component. Because of the near mass degeneracy, the charged scalar can lead to signatures in both disappearing tracks and searches for heavy stably charged particles at the LHC. These signatures are however very much dependent on the exact amount of compression within the components of the inert doublet. In this case, we also illustrated the complementarity of current and future direct and indirect detection searches and provided benchmarks to that effect. We showed that while most of the parameter space could be probed in the future, some scenarios escape detection. As in the general case, they correspond to cases where the couplings of DM to the Higgs is suppressed, and there is a significant amount of semiannihilation or DM conversion. Because semiannihilation often leads to a much softer photon spectrum in indirect detection, improving the sensitivity at low energies would be required to probe all scenarios. In particular, we found that when DM conversion was important, a pair of doublets could annihilate into a pair of singlets if kinematically accessible rendering both DM invisible.

In this paper, we considered only the case where the singlet and the doublet are WIMPs; in general, in this model, the couplings of the singlet can be so weak that it is feebly interacting and is produced via freeze-in in the early Universe. A detailed analysis of this case is left for a separate analysis.

\section{ACKNOWLEDGMENTS}

We thank C. Eckner for helpful discussions on CTA and the use of his code for reading the spectrum tables. We thank
Shankha Banerjee for checking the one-loop contribution to the charged Higgs partial decay width. We also acknowledge useful discussions with Alexander Belyaev, Fawzi Boudjema, Francesca Calore, Bjorn Herrmann, and Sabine Kraml. This work was funded by RFBR and CNRS, Project No. 20-5215005. The work of A. Pukhov was also supported in part by Grant No. AAP-USMB and by the Interdisciplinary Scientific and Educational School of Moscow University Fundamental and Applied Space Research.

\section{APPENDIX A: GENERAL SCAN}

Here, we summarize the contributions to DM annihilation from the different final states as found in the general scan. We compute $\left\langle v \sigma^{\alpha \beta \gamma \delta}\right\rangle$ for which we use a shorthand notation $\langle v \sigma\rangle_{\gamma \delta}$ to represent the contribution of a given initial state of DM particles in the channel $\gamma \delta$ to the total annihilation cross section. This quantity implicitly takes into account the fraction of the DM abundance for each DM candidate. In the corresponding figures, we display only final states for which the cross section can reach at least $10^{-27} \mathrm{~cm}^{3} / \mathrm{s}$ since this is already below the sensitivity of Fermi-LAT and even of the future CTA.

Despite the fact that $\tilde{A}$ is the subdominant DM component, the indirect detection signal from pair annihilation into $W(Z)$ pairs can be dominant, and it can reach $10^{-25} \mathrm{~cm}^{3} / \mathrm{s}$, see Fig. 21. Moreover, $\langle v \sigma\rangle_{W W / Z Z}$ can exceed $10^{-26} \mathrm{~cm}^{3} / \mathrm{s}$ in almost the full range of $M_{A}$ considered. The region where the cross section is suppressed corresponds to the one below roughly $100 \mathrm{GeV}$. Note also that in many cases the $Z Z$ channel is much larger than the $W W$ channel. This peculiar behavior is related to the fact that when the doublet is compressed there is a cancellation between the quartic diagram and the t-channel diagram shown in Fig. 1, leading to a much smaller cross section. Since the mass splitting $\Delta^{+}$can be much smaller than $\Delta^{0}$, as seen in Fig. 22, the cross section in the $W W$ channel can be much suppressed relative to the one in the $Z Z$ channel. The main subdominant contribution is from the $h h$ channel, the largest value being $\langle v \sigma\rangle_{h h}=4 \times 10^{-27} \mathrm{~cm}^{3} / \mathrm{s}$, while, in general, the quark channels $(t \bar{t}$ or $b \bar{b}$ ) are suppressed by many orders of magnitude. For example, the latter reaches at most $4 \times 10^{-28} \mathrm{~cm}^{3} / \mathrm{s}$.

The annihilation cross section for the dominant DM component, $\tilde{S}$, into pairs of SM particles is much smaller than for the doublet; it can reach only $7 \times 10^{-27} \mathrm{~cm}^{3} / \mathrm{s}$. The channels with larger cross sections are $W W, Z Z$, and to a lesser extent $h h$, while $t \bar{t}$ and $b \bar{b}$ are negligible, lying below $10^{-28} \mathrm{~cm}^{3} / \mathrm{s}$. Interactions between the two DM components often provide the dominant semiannihilation channels for the singlet. For semiannihilation processes such as $\tilde{S} \tilde{S} \rightarrow \tilde{H}^{-} W^{+}$or $\tilde{H}^{+} W^{-}$, the cross section is typically concentrated near $10^{-26} \mathrm{~cm}^{3} / \mathrm{s}$ for any value of $M_{S}$ and can in a few cases reach $7 \times 10^{-26} \mathrm{~cm}^{3} / \mathrm{s}$. Other channels 

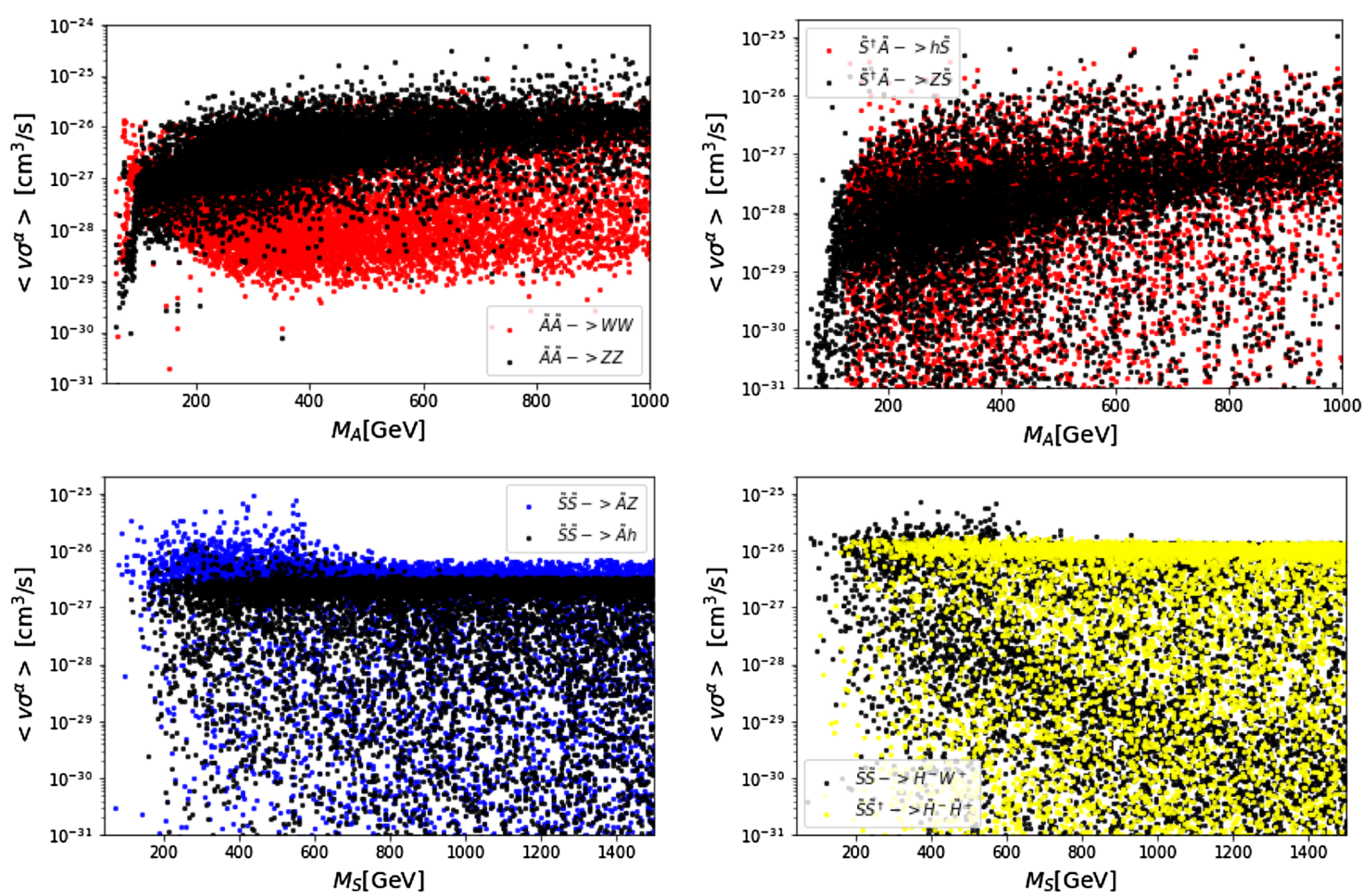

FIG. 21. Cross section, $\left\langle v \sigma^{\alpha}\right\rangle$ for the channels that lead to the largest cross sections. The channels $\alpha$ are specified in each panel.
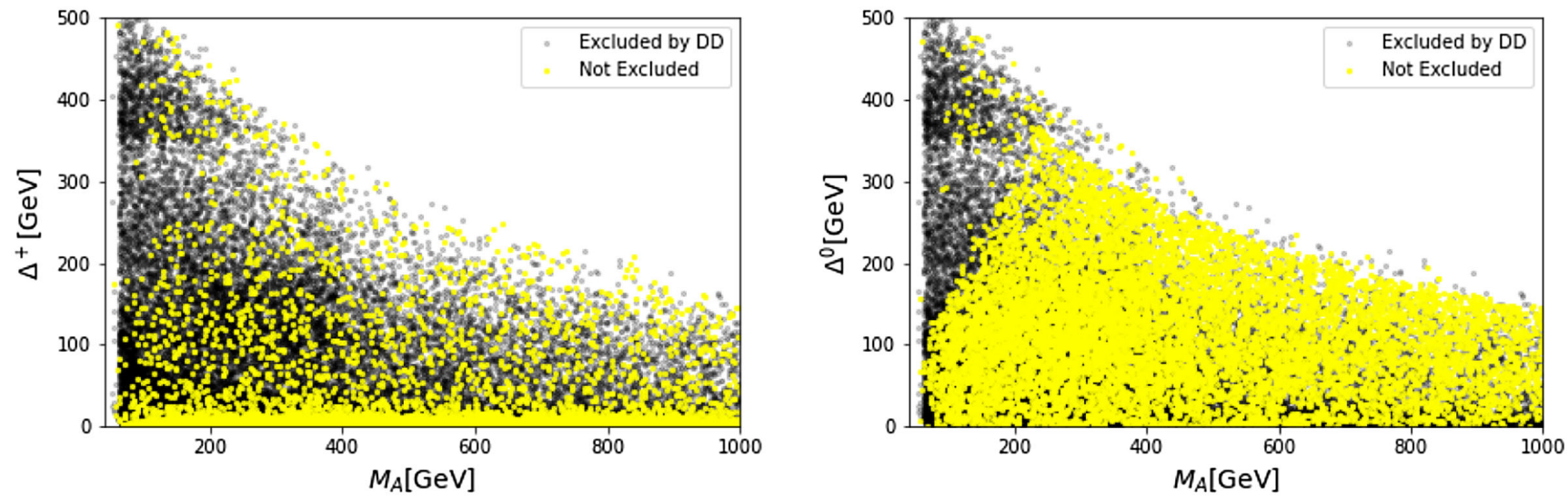

FIG. 22. Allowed points in the $\Delta^{+}-M_{A}$ plane (left) and $\Delta^{0}-M_{A}$ plane (right), same color code as Fig. 8 .

where a pair of singlets DM annihilates into a doublet component and a standard model, such as $\tilde{S} \tilde{S} \rightarrow \tilde{A} h, \tilde{A} Z$ can exceed $10^{-26} \mathrm{~cm}^{3} / \mathrm{s}$ especially for $M_{S}<500 \mathrm{GeV}$. Note that $\tilde{S} \tilde{S} \rightarrow \tilde{H} h, \tilde{H} Z$ lead to similar cross sections after having taken into account the heavier mass of $\tilde{H}$. Semiannihilation processes such as $\tilde{S}^{\dagger} \tilde{A} \rightarrow h \tilde{S}, Z \tilde{S}$ lie generally below $10^{-26} \mathrm{~cm}^{3} / \mathrm{s}$ except for a few points.
The DM conversion processes can also be important; notably, $\tilde{S} \tilde{S} \rightarrow \tilde{H}^{+} \tilde{H}^{-}$for which $\left\langle\sigma_{v}\right\rangle_{H^{+} H^{-}}$is generally around $10^{-26} \mathrm{~cm}^{3} / \mathrm{s}$ for any masses, while $\tilde{S} \tilde{S} \rightarrow \tilde{H} \tilde{H}$ is slightly suppressed. Finally, other DM conversion processes such as $\tilde{A} \tilde{A} \rightarrow \tilde{S} \tilde{S}$ or the reverse process can be large, reaching above $10^{-25} \mathrm{~cm}^{3} / \mathrm{s}$; however, these modes are invisible and do not contribute to the signal. 

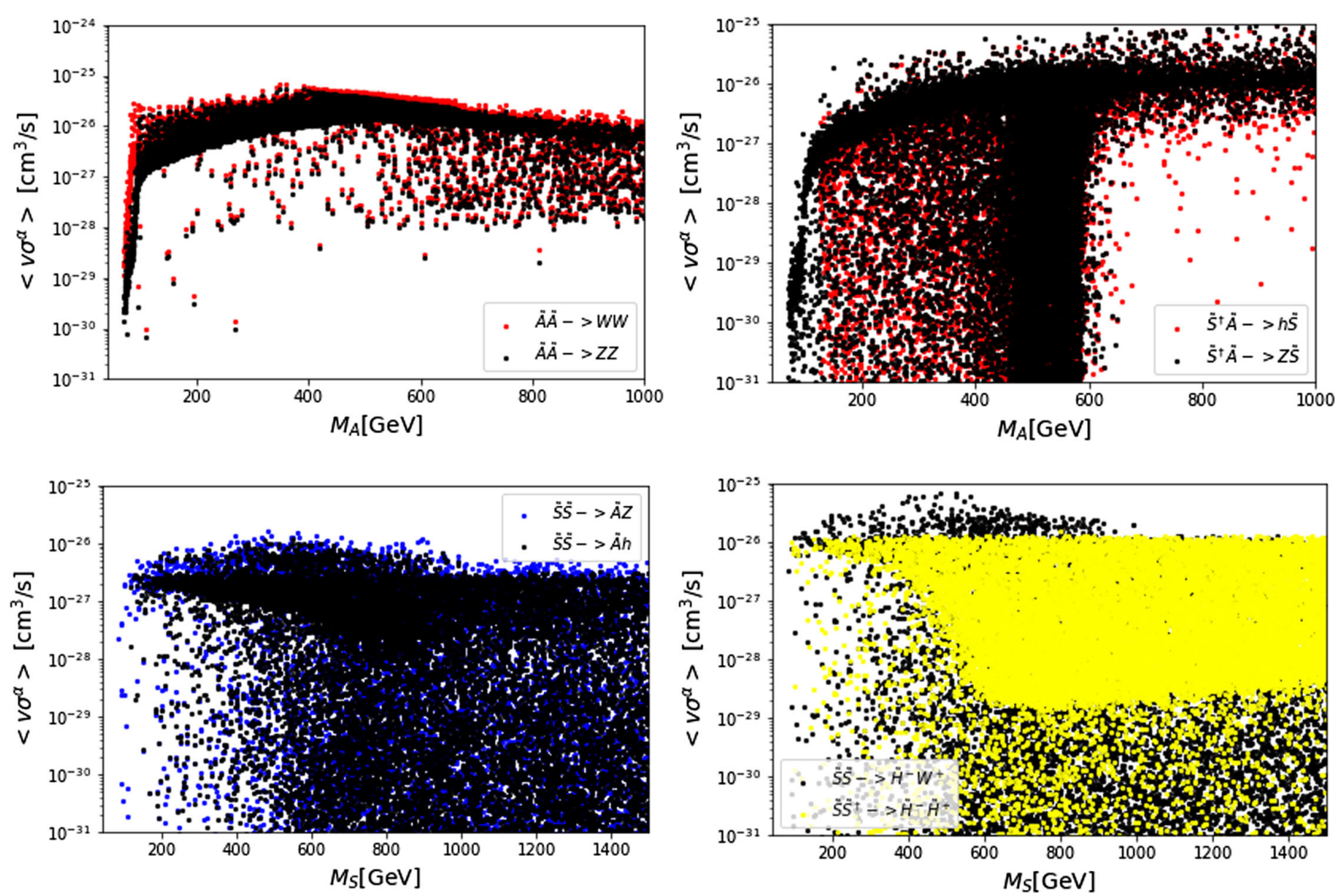

FIG. 23. Cross sections for the main DM annihilation and semiannihilation channels indicated in each panel.

\section{APPENDIX B: NEARLY DEGENERATE DOUBLET}

The largest annihilation cross sections for the doublet are into $W W / Z Z$ pairs; they can reach $\left(\approx 10^{-25} \mathrm{~cm}^{3} / \mathrm{s}\right)$ when $M_{A} \approx 400 \mathrm{GeV}$ as shown in Fig. 23. In this case, the doublet forms the dominant DM component. Typically, the $W W$ channel is slightly larger than the $Z Z$ channel since all doublet components are nearly degenerate. As in the noncompressed case, other SM final states are subdominant. The only other important annihilation channel for the doublets are into pairs of singlets, and although this cross section can exceed $10^{-25} \mathrm{~cm}^{3} / \mathrm{s}$, it does not contribute to the signal.

The pair annihilation channels $\tilde{S} \tilde{S} \rightarrow S M, S M$ reach at most $7 \times 10^{-27} \mathrm{~cm}^{3} / \mathrm{s}$ in the $W W$ channel. As in the general case, other final states that can exceed $10^{-26} \mathrm{~cm}^{3} / \mathrm{s}$ include most of the semiannihilation $\tilde{S} \tilde{S}^{\dagger} \rightarrow \tilde{H}^{ \pm} W^{\mp}, \tilde{A} h$, $\tilde{A} Z, \tilde{H} h, \tilde{H} Z$ or DM conversion processes $\tilde{S} \tilde{S}^{\dagger} \rightarrow \tilde{H}^{+} \tilde{H}^{-}$. Moreover, semiannihilation channels such as $\tilde{S}^{\dagger} \tilde{A} \rightarrow h \tilde{S}$, $Z \tilde{S}$ can be dominant, and both can reach approximately $10^{-25} \mathrm{~cm}^{3} / \mathrm{s}$ for $M_{A}>600 \mathrm{GeV}$.
[1] E. Aprile et al. (XENON Collaboration), Dark Matter Search Results from a One Tonne $\times$ Year Exposure of XENON1T, Phys. Rev. Lett. 121, 111302 (2018).

[2] M. Ackermann et al. (Fermi-LAT Collaboration), Searching for Dark Matter Annihilation from Milky Way Dwarf Spheroidal Galaxies with Six Years of Fermi
Large Area Telescope Data, Phys. Rev. Lett. 115, 231301 (2015).

[3] A. Albert et al. (Fermi-LAT, DES Collaborations), Searching for dark matter annihilation in recently discovered Milky Way satellites with Fermi-LAT, Astrophys. J. 834, 110 (2017). 
[4] A. Albert et al. (HAWC Collaboration), Search for gammaray spectral lines from dark matter annihilation in dwarf galaxies with the high-altitude water Cherenkov observatory, Phys. Rev. D 101, 103001 (2020).

[5] D. Abercrombie et al., Dark matter benchmark models for early LHC run-2 searches: Report of the ATLAS/CMS dark matter forum, Phys. Dark Universe 27, 100371 (2020).

[6] Q. Wang et al. (PandaX-II Collaboration), Results of dark matter search using the full PandaX-II exposure, Chin. Phys. C 44, 125001 (2020).

[7] C. Amole et al. (PICO Collaboration), Dark matter search results from the complete exposure of the PICO-60 $\mathrm{C}_{3} \mathrm{~F}_{8}$ bubble chamber, Phys. Rev. D 100, 022001 (2019).

[8] L. Oakes et al. (Fermi-LAT, HAWC, H.E.S.S., MAGIC, VERITAS Collaborations), Combined dark matter searches towards dwarf spheroidal galaxies with FermiLAT, HAWC, HESS, MAGIC and VERITAS, Proc. Sci. ICRC2019 (2021) 012 [arXiv:1909.06310].

[9] A. Acharyya et al. (CTA Collaboration), Sensitivity of the cherenkov telescope array to a dark matter signal from the galactic centre, J. Cosmol. Astropart. Phys. 01 (2021) 057.

[10] L. Roszkowski, E. M. Sessolo, and S. Trojanowski, WIMP dark matter candidates and searches-current status and future prospects, Rep. Prog. Phys. 81, 066201 (2018).

[11] G. Arcadi, M. Dutra, P. Ghosh, M. Lindner, Y. Mambrini, M. Pierre, S. Profumo, and F. S. Queiroz, The waning of the WIMP? A review of models, searches, and constraints, Eur. Phys. J. C 78, 203 (2018).

[12] G. Bertone and T. M. P. Tait, A new era in the search for dark matter, Nature (London) 562, 51 (2018).

[13] S. Knapen, T. Lin, and K. M. Zurek, Light dark matter: Models and constraints, Phys. Rev. D 96, 115021 (2017).

[14] M. Cirelli, Y. Gouttenoire, K. Petraki, and F. Sala, Homeopathic dark matter, or how diluted heavy substances produce high energy cosmic rays, J. Cosmol. Astropart. Phys. 02 (2019) 014.

[15] J. McDonald, Gauge singlet scalars as cold dark matter, Phys. Rev. D 50, 3637 (1994).

[16] L. J. Hall, K. Jedamzik, J. March-Russell, and S. M. West, Freeze-in production of FIMP dark matter, J. High Energy Phys. 03 (2010) 080.

[17] N. Bernal, M. Heikinheimo, T. Tenkanen, K. Tuominen, and V. Vaskonen, The dawn of FIMP dark matter: A review of models and constraints, Int. J. Mod. Phys. A 32, 1730023 (2017).

[18] Y. Hochberg, E. Kuflik, T. Volansky, and J. G. Wacker, Mechanism for Thermal Relic Dark Matter of Strongly Interacting Massive Particles, Phys. Rev. Lett. 113, 171301 (2014).

[19] Y. Hochberg, E. Kuflik, H. Murayama, T. Volansky, and J. G. Wacker, Model for Thermal Relic Dark Matter of Strongly Interacting Massive Particles, Phys. Rev. Lett. 115, 021301 (2015).

[20] H. M. Lee and M.-S. Seo, Communication with SIMP dark mesons via Z' -portal, Phys. Lett. B 748, 316 (2015).

[21] J. Smirnov and J. F. Beacom, New Freezeout Mechanism for Strongly Interacting Dark Matter, Phys. Rev. Lett. 125, 131301 (2020).

[22] T. Asaka and T. Yanagida, Solving the gravitino problem by axino, Phys. Lett. B 494, 297 (2000).
[23] L. Covi, L. Roszkowski, R. Ruiz de Austri, and M. Small, Axino dark matter and the CMSSM, J. High Energy Phys. 06 (2004) 003.

[24] H. Baer, R. Dermisek, S. Rajagopalan, and H. Summy, Neutralino, axion and axino cold dark matter in minimal, hypercharged and gaugino AMSB, J. Cosmol. Astropart. Phys. 07 (2010) 014.

[25] K. J. Bae, H. Baer, and E. J. Chun, Mixed axion/neutralino dark matter in the SUSY DFSZ axion model, J. Cosmol. Astropart. Phys. 12 (2013) 028.

[26] L. Di Luzio, M. Giannotti, E. Nardi, and L. Visinelli, The landscape of QCD axion models, Phys. Rep. 870, 1 (2020).

[27] I. G. Irastorza and J. Redondo, New experimental approaches in the search for axion-like particles, Prog. Part. Nucl. Phys. 102, 89 (2018).

[28] P. Agrawal et al., Feebly-interacting particles: FIPs 2020 workshop report, Eur. Phys. J. C 81, 1015 (2021).

[29] G. Belanger and J.-C. Park, Assisted freeze-out, J. Cosmol. Astropart. Phys. 03 (2012) 038.

[30] G. Belanger, K. Kannike, A. Pukhov, and M. Raidal, Impact of semi-annihilations on dark matter phenomenology-an example of $Z_{N}$ symmetric scalar dark matter, J. Cosmol. Astropart. Phys. 04 (2012) 010.

[31] G. Bélanger, A. Pukhov, C. E. Yaguna, and O. Zapata, The $\mathrm{Z}_{5}$ model of two-component dark matter, J. High Energy Phys. 09 (2020) 030.

[32] T. Hambye, Hidden vector dark matter, J. High Energy Phys. 01 (2009) 028.

[33] T. Hambye and M. H. G. Tytgat, Confined hidden vector dark matter, Phys. Lett. B 683, 39 (2010).

[34] F. D'Eramo and J. Thaler, Semi-annihilation of dark matter, J. High Energy Phys. 06 (2010) 109.

[35] G. Arcadi, C. Gross, O. Lebedev, Y. Mambrini, S. Pokorski, and T. Toma, Multicomponent dark matter from gauge symmetry, J. High Energy Phys. 12 (2016) 081.

[36] T. N. Maity and T. S. Ray, Exchange driven freeze out of dark matter, Phys. Rev. D 101, 103013 (2020).

[37] A. Drozd, B. Grzadkowski, and J. Wudka, Multi-scalarsinglet extension of the standard model - the case for dark matter and an invisible Higgs boson, J. High Energy Phys. 04 (2012) 006.

[38] A. Drozd, B. Grzadkowski, and J. Wudka, Cosmology of multi-singlet-scalar extensions of the standard model, Acta Phys. Pol. B 42, 2255 (2011).

[39] A. Biswas, D. Majumdar, A. Sil, and P. Bhattacharjee, Two component dark matter: A possible explanation of $130 \mathrm{GeV} \gamma$ - ray line from the galactic centre, J. Cosmol. Astropart. Phys. 12 (2013) 049.

[40] S. Bhattacharya, P. Poulose, and P. Ghosh, Multipartite interacting scalar dark matter in the light of updated LUX data, J. Cosmol. Astropart. Phys. 04 (2017) 043.

[41] S. Bhattacharya, P. Ghosh, T. N. Maity, and T. S. Ray, Mitigating direct detection bounds in non-minimal Higgs portal scalar dark matter models, J. High Energy Phys. 10 (2017) 088.

[42] N. Chakrabarty, R. Roshan, and A. Sil, Two component doublet-triplet scalar dark matter stabilising the electroweak vacuum, arXiv:2102.06032. 
[43] M. Cirelli and J. M. Cline, Can multistate dark matter annihilation explain the high-energy cosmic ray lepton anomalies?, Phys. Rev. D 82, 023503 (2010).

[44] J. Heeck and H. Zhang, Exotic charges, multicomponent dark matter and light sterile neutrinos, J. High Energy Phys. 05 (2013) 164.

[45] Y. Kajiyama, H. Okada, and T. Toma, Multicomponent dark matter particles in a two-loop neutrino model, Phys. Rev. D 88, 015029 (2013).

[46] P.-H. Gu, Multi-component dark matter with magnetic moments for Fermi-LAT gamma-ray line, Phys. Dark Universe 2, 35 (2013).

[47] N. Bernal, D. Restrepo, C. Yaguna, and O. Zapata, Twocomponent dark matter and a massless neutrino in a new B-L model, Phys. Rev. D 99, 015038 (2019).

[48] C. Gross, O. Lebedev, and Y. Mambrini, Non-Abelian gauge fields as dark matter, J. High Energy Phys. 08 (2015) 158 .

[49] A. Karam and K. Tamvakis, Dark matter from a classically scale-invariant $S U(3)_{X}$, Phys. Rev. D 94, 055004 (2016).

[50] Y. Daikoku, H. Okada, and T. Toma, Two component dark matters in $S_{4} \times Z_{2}$ flavor symmetric extra U(1) model, Prog. Theor. Phys. 126, 855 (2011).

[51] Y. Farzan and A. R. Akbarieh, VDM: A model for vector dark matter, J. Cosmol. Astropart. Phys. 10 (2012) 026.

[52] S. Bhattacharya, A. Drozd, B. Grzadkowski, and J. Wudka, Two-component dark matter, J. High Energy Phys. 10 (2013) 158.

[53] L. Bian, R. Ding, and B. Zhu, Two component Higgsportal dark matter, Phys. Lett. B 728, 105 (2014).

[54] S. Esch, M. Klasen, and C. E. Yaguna, A minimal model for two-component dark matter, J. High Energy Phys. 09 (2014) 108.

[55] A. DiFranzo and G. Mohlabeng, Multi-component dark matter through a radiative Higgs portal, J. High Energy Phys. 01 (2017) 080.

[56] A. Ahmed, M. Duch, B. Grzadkowski, and M. Iglicki, Multi-component dark matter: The vector and fermion case, Eur. Phys. J. C 78, 905 (2018).

[57] S. Chakraborti and P. Poulose, Interplay of scalar and fermionic components in a multi-component dark matter scenario, Eur. Phys. J. C 79, 420 (2019).

[58] A. Poulin and S. Godfrey, Multicomponent dark matter from a hidden gauged SU(3), Phys. Rev. D 99, 076008 (2019).

[59] S. Profumo, K. Sigurdson, and L. Ubaldi, Can we discover multi-component WIMP dark matter?, J. Cosmol. Astropart. Phys. 12 (2009) 016.

[60] K. M. Zurek, Multi-component dark matter, Phys. Rev. D 79, 115002 (2009).

[61] F. Elahi and S. Khatibi, Multi-component dark matter in a non-abelian dark sector, Phys. Rev. D 100, 015019 (2019).

[62] S. Khalil, S. Moretti, D. Rojas-Ciofalo, and H. Waltari, Multicomponent dark matter in a simplified $\mathrm{E}_{6} \mathrm{SSM}$, Phys. Rev. D 102, 075039 (2020).

[63] H. S. Cheon, S. K. Kang, and C. S. Kim, Doubly coexisting dark matter candidates in an extended seesaw model, Phys. Lett. B 675, 203 (2009).
[64] D. Borah, R. Roshan, and A. Sil, Minimal two-component scalar doublet dark matter with radiative neutrino mass, Phys. Rev. D 100, 055027 (2019).

[65] A. Biswas, D. Borah, and D. Nanda, Type III seesaw for neutrino masses in $U(1)_{B-L}$ model with multi-component dark matter, J. High Energy Phys. 12 (2019) 109.

[66] S. Bhattacharya, P. Ghosh, A. K. Saha, and A. Sil, Two component dark matter with inert Higgs doublet: Neutrino mass, high scale validity and collider searches, J. High Energy Phys. 03 (2020) 090.

[67] B. Batell, Dark discrete gauge symmetries, Phys. Rev. D 83, 035006 (2011).

[68] I. P. Ivanov and V. Keus, $Z_{p}$ scalar dark matter from multiHiggs-doublet models, Phys. Rev. D 86, 016004 (2012).

[69] G. Bélanger, K. Kannike, A. Pukhov, and M. Raidal, Minimal semi-annihilating $\mathbb{Z}_{N}$ scalar dark matter, J. Cosmol. Astropart. Phys. 06 (2014) 021.

[70] C. E. Yaguna and O. Zapata, Multi-component scalar dark matter from a $Z_{N}$ symmetry: A systematic analysis, J. High Energy Phys. 03 (2020) 109.

[71] A. Aranda, D. Hernández-Otero, J. Hernández-Sanchez, V. Keus, S. Moretti, D. Rojas-Ciofalo, and T. Shindou, $\mathrm{Z}_{3}$ symmetric inert $(2+1)$-Higgs-doublet model, Phys. Rev. D 103, 015023 (2021).

[72] M. Malekjani, S. Rahvar, and D. M.Z. Jassur, Two component baryonic-dark matter structure formation in top-hat model, New Astron. 14, 398 (2009).

[73] V. Semenov, S. Pilipenko, A. Doroshkevich, V. Lukash, and E. Mikheeva, Dark matter halo formation in the multicomponent dark matter models, arXiv:1306.3210.

[74] M. V. Medvedev, Cosmological Simulations of Multicomponent Cold Dark Matter, Phys. Rev. Lett. 113, 071303 (2014).

[75] M. Rocha, A. H. G. Peter, J. S. Bullock, M. Kaplinghat, S. Garrison-Kimmel, J. Onorbe, and L. A. Moustakas, Cosmological simulations with self-interacting dark matter I: Constant density cores and substructure, Mon. Not. R. Astron. Soc. 430, 81 (2013).

[76] D. H. Weinberg, J. S. Bullock, F. Governato, R. Kuzio de Naray, and A. H. G. Peter, Cold dark matter: Controversies on small scales, Proc. Natl. Acad. Sci. U.S.A. 112, 12249 (2015).

[77] D. N. Spergel and P. J. Steinhardt, Observational Evidence for Selfinteracting Cold Dark Matter, Phys. Rev. Lett. 84, 3760 (2000).

[78] W. B. Atwood et al. (Fermi-LAT Collaboration), The large area telescope on the fermi gamma-ray space telescope mission, Astrophys. J. 697, 1071 (2009).

[79] F. Calore, I. Cholis, C. McCabe, and C. Weniger, A tale of tails: Dark matter interpretations of the fermi gev excess in light of background model systematics, Phys. Rev. D 91, 063003 (2015).

[80] N. G. Deshpande and E. Ma, Pattern of symmetry breaking with two Higgs doublets, Phys. Rev. D 18, 2574 (1978).

[81] R. Barbieri, L. J. Hall, and V.S. Rychkov, Improved naturalness with a heavy Higgs: An alternative road to LHC physics, Phys. Rev. D 74, 015007 (2006).

[82] L. Lopez Honorez, E. Nezri, J. F. Oliver, and M. H. G. Tytgat, The inert doublet model: An archetype for dark matter, J. Cosmol. Astropart. Phys. 02 (2007) 028. 
[83] A. Urbano and W. Xue, Constraining the Higgs portal with antiprotons, J. High Energy Phys. 03 (2015) 133.

[84] L. Feng, S. Profumo, and L. Ubaldi, Closing in on singlet scalar dark matter: LUX, invisible Higgs decays and gamma-ray lines, J. High Energy Phys. 03 (2015) 045.

[85] A. Goudelis, Y. Mambrini, and C. Yaguna, Antimatter signals of singlet scalar dark matter, J. Cosmol. Astropart. Phys. 12 (2009) 008.

[86] C. E. Yaguna, Gamma rays from the annihilation of singlet scalar dark matter, J. Cosmol. Astropart. Phys. 03 (2009) 003.

[87] H. Han, J. M. Yang, Y. Zhang, and S. Zheng, Collider signatures of Higgs-portal scalar dark matter, Phys. Lett. B 756, 109 (2016).

[88] Y. Mambrini, Higgs searches and singlet scalar dark matter: Combined constraints from XENON 100 and the LHC, Phys. Rev. D 84, 115017 (2011).

[89] P. A. R. Ade et al. (Planck Collaboration), Planck 2015 results. XIII. Cosmological parameters, Astron. Astrophys. 594, A13 (2016).

[90] N. Aghanim et al. (Planck Collaboration), Planck 2018 results. VI. Cosmological parameters, Astron. Astrophys. 641, A6 (2020).

[91] K. Cheung, Y.-L. S. Tsai, P.-Y. Tseng, T.-C. Yuan, and A. Zee, Global study of the simplest scalar phantom dark matter model, J. Cosmol. Astropart. Phys. 10 (2012) 042.

[92] J. M. Cline, K. Kainulainen, P. Scott, and C. Weniger, Update on scalar singlet dark matter, Phys. Rev. D 88, 055025 (2013); 92, 039906(E) (2015).

[93] P. Athron et al. (GAMBIT Collaboration), Status of the scalar singlet dark matter model, Eur. Phys. J. C 77, 568 (2017).

[94] P. Athron, J. M. Cornell, F. Kahlhoefer, J. Mckay, P. Scott, and S. Wild, Impact of vacuum stability, perturbativity and XENON1T on global fits of $\mathbb{Z}_{2}$ and $\mathbb{Z}_{3}$ scalar singlet dark matter, Eur. Phys. J. C 78, 830 (2018).

[95] P. Athron et al. (GAMBIT Collaboration), Global analyses of Higgs portal singlet dark matter models using GAMBIT, Eur. Phys. J. C 79, 38 (2019).

[96] A. Belyaev, G. Cacciapaglia, I. P. Ivanov, F. Rojas-Abatte, and M. Thomas, Anatomy of the inert two Higgs doublet model in the light of the LHC and non-LHC dark matter searches, Phys. Rev. D 97, 035011 (2018).

[97] A. Goudelis, B. Herrmann, and O. Stål, Dark matter in the inert doublet model after the discovery of a Higgs-like boson at the LHC, J. High Energy Phys. 09 (2013) 106.

[98] A. Arhrib, Y.-L. S. Tsai, Q. Yuan, and T.-C. Yuan, An updated analysis of inert Higgs doublet model in light of the recent results from LUX, PLANCK, AMS-02 and LHC, J. Cosmol. Astropart. Phys. 06 (2014) 030.

[99] M. A. Díaz, B. Koch, and S. Urrutia-Quiroga, Constraints to dark matter from inert Higgs doublet model, Adv. High Energy Phys. 2016, 8278375 (2016).

[100] B. Eiteneuer, A. Goudelis, and J. Heisig, The inert doublet model in the light of Fermi-LAT gamma-ray data: A global fit analysis, Eur. Phys. J. C 77, 624 (2017).

[101] K. P. Modak and D. Majumdar, Confronting galactic and extragalactic $\gamma$-rays observed by fermi-lat with annihilating dark matter in an inert Higgs doublet model, Astrophys. J. Suppl. Ser. 219, 37 (2015).
[102] F. S. Queiroz and C. E. Yaguna, The CTA aims at the inert doublet model, J. Cosmol. Astropart. Phys. 02 (2016) 038.

[103] C. Garcia-Cely, M. Gustafsson, and A. Ibarra, Probing the inert doublet dark matter model with cherenkov telescopes, J. Cosmol. Astropart. Phys. 02 (2016) 043.

[104] M. Krawczyk, D. Sokolowska, P. Swaczyna, and B. Swiezewska, Constraining inert dark matter by $R_{\gamma \gamma}$ and WMAP data, J. High Energy Phys. 09 (2013) 055.

[105] G. Belanger, B. Dumont, A. Goudelis, B. Herrmann, S. Kraml, and D. Sengupta, Dilepton constraints in the inert doublet model from run 1 of the LHC, Phys. Rev. D 91, 115011 (2015).

[106] X. Miao, S. Su, and B. Thomas, Trilepton signals in the inert doublet model, Phys. Rev. D 82, 035009 (2010).

[107] M. Hashemi and S. Najjari, Observability of inert scalars at the LHC, Eur. Phys. J. C 77, 592 (2017).

[108] M. Gustafsson, S. Rydbeck, L. Lopez-Honorez, and E. Lundstrom, Status of the inert doublet model and the role of multileptons at the LHC, Phys. Rev. D 86, 075019 (2012).

[109] P. Poulose, S. Sahoo, and K. Sridhar, Exploring the inert doublet model through the dijet plus missing transverse energy channel at the LHC, Phys. Lett. B 765, 300 (2017).

[110] A. Ilnicka, M. Krawczyk, and T. Robens, Inert doublet model in light of LHC run I and astrophysical data, Phys. Rev. D 93, 055026 (2016).

[111] N. Blinov, J. Kozaczuk, D. E. Morrissey, and A. de la Puente, Compressing the inert doublet model, Phys. Rev. D 93, 035020 (2016).

[112] E. Aprile et al. (XENON Collaboration), Physics reach of the XENON1T dark matter experiment, J. Cosmol. Astropart. Phys. 04 (2016) 027.

[113] J. Aalbers et al. (DARWIN Collaboration), DARWIN: Towards the ultimate dark matter detector, J. Cosmol. Astropart. Phys. 11 (2016) 017.

[114] C. H. Chen, M. Drees, and J. F. Gunion, A nonstandard string / SUSY scenario and its phenomenological implications, Phys. Rev. D 55, 330 (1997).

[115] A. Belyaev, S. Prestel, F. Rojas-Abbate, and J. Zurita, Probing dark matter with disappearing tracks at the LHC, Phys. Rev. D 103, 095006 (2021).

[116] M. D. Goodsell, S. Kraml, H. Reyes-González, and S. L. Williamson, Constraining electroweakinos in the minimal dirac gaugino model, SciPost Phys. 9, 047 (2020).

[117] A. Mjallal, Ph.D. thesis USMB (2021).

[118] S. Banerjee, F. Boudjema, N. Chakrabarty, G. Chalons, and H. Sun, Relic density of dark matter in the inert doublet model beyond leading order: The heavy mass case, Phys. Rev. D 100, 095024 (2019).

[119] S. Banerjee, F. Boudjema, N. Chakrabarty, and H. Sun, Relic density of dark matter in the inert doublet model beyond leading order for the low mass region: 2. Co-annihilation, Phys. Rev. D 104, 075003 (2021).

[120] S. Banerjee, F. Boudjema, N. Chakrabarty, and H. Sun, Relic density of dark matter in the inert doublet model beyond leading order for the low mass region: 3. Annihilation in 3-body final state, Phys. Rev. D 104, 075004 (2021).

[121] S. Banerjee, F. Boudjema, N. Chakrabarty, and H. Sun, Relic density of dark matter in the inert doublet model beyond leading order for the low mass region: 4 . The Higgs resonance region, Phys. Rev. D 104, 075005 (2021). 
[122] S. Banerjee, F. Boudjema, N. Chakrabarty, and H. Sun, Relic density of dark matter in the inert doublet model beyond leading order for the low mass region: 1. Renormalisation and constraints, Phys. Rev. D 104, 075002 (2021).

[123] S. Banerjee (private communication).

[124] R. N. Lerner and J. McDonald, Gauge singlet scalar as inflaton and thermal relic dark matter, Phys. Rev. D 80, 123507 (2009).

[125] K. Kannike, Vacuum stability conditions from copositivity criteria, Eur. Phys. J. C 72, 2093 (2012).

[126] M. Baak, J. Cúth, J. Haller, A. Hoecker, R. Kogler, K. Mönig, M. Schott, and J. Stelzer (Gfitter Group Collaboration), The global electroweak fit at NNLO and prospects for the LHC and ILC, Eur. Phys. J. C 74, 3046 (2014).

[127] E. Lundstrom, M. Gustafsson, and J. Edsjo, The inert doublet model and LEP II limits, Phys. Rev. D 79, 035013 (2009).

[128] A. Pierce and J. Thaler, Natural dark matter from an unnatural Higgs boson and new colored particles at the TeV scale, J. High Energy Phys. 08 (2007) 026.

[129] G. Abbiendi et al. (OPAL Collaboration), Search for stable and longlived massive charged particles in $e+e-$ collisions at $\mathrm{s}^{* *}(1 / 2)=130-\mathrm{GeV}$ to $209-\mathrm{GeV}$, Phys. Lett. B 572, 8 (2003).

[130] A. M. Sirunyan et al. (CMS Collaboration), Search for invisible decays of a Higgs boson produced through vector boson fusion in proton-proton collisions at $\sqrt{s}=13 \mathrm{TeV}$, Phys. Lett. B 793, 520 (2019).

[131] ATLAS Collaboration, Report No. ATLAS-CONF-2020052.

[132] G. Belanger, F. Boudjema, A. Pukhov, and A. Semenov, micrOMEGAs_3: A program for calculating dark matter observables, Comput. Phys. Commun. 185, 960 (2014).

[133] P. Bechtle, S. Heinemeyer, T. Klingl, T. Stefaniak, G. Weiglein, and J. Wittbrodt, HiggsSignals-2: Probing new physics with precision Higgs measurements in the LHC 13 TeV era, Eur. Phys. J. C 81, 145 (2021).

[134] A. Belyaev, T. R. Fernandez Perez Tomei, P. G. Mercadante, C. S. Moon, S. Moretti, S. F. Novaes, L. Panizzi, F. Rojas, and M. Thomas, Advancing LHC probes of dark matter from the inert two-Higgs-doublet model with the monojet signal, Phys. Rev. D 99, 015011 (2019).

[135] D. Dercks and T. Robens, Constraining the inert doublet model using vector boson fusion, Eur. Phys. J. C 79, 924 (2019).

[136] M. Aaboud et al. (ATLAS Collaboration), Search for dark matter and other new phenomena in events with an energetic jet and large missing transverse momentum using the ATLAS detector, J. High Energy Phys. 01 (2018) 126.

[137] M. Drees, H. Dreiner, D. Schmeier, J. Tattersall, and J. S. Kim, CheckMATE: Confronting your favourite new physics model with LHC data, Comput. Phys. Commun. 187, 227 (2015).

[138] D. Dercks, N. Desai, J. S. Kim, K. Rolbiecki, J. Tattersall, and T. Weber, CheckMATE 2: From the model to the limit, Comput. Phys. Commun. 221, 383 (2017).

[139] F. Ambrogi et al., SModelS v1.2: Long-lived particles, combination of signal regions, and other novelties, Comput. Phys. Commun. 251, 106848 (2020).
[140] C. K. Khosa, S. Kraml, A. Lessa, P. Neuhuber, and W. Waltenberger, SModelS database update v1.2.3, Lett. High Energy Phys. 2020, 158 (2020).

[141] G. Alguero, S. Kraml, and W. Waltenberger, A Smodels interface for PYHF likelihoods, Comput. Phys. Commun. 264, 107909 (2021).

[142] V. Khachatryan et al. (CMS Collaboration), Constraints on the pMSSM, AMSB model and on other models from the search for long-lived charged particles in proton-proton collisions at $\sqrt{s}=8 \mathrm{TeV}$, Eur. Phys. J. C 75, 325 (2015).

[143] M. Aaboud et al. (ATLAS Collaboration), Search for heavy charged long-lived particles in the ATLAS detector in $36.1 \mathrm{fb}^{-1}$ of proton-proton collision data at $\sqrt{\mathrm{s}}=$ 13 TeV, Phys. Rev. D 99, 092007 (2019).

[144] J. Heisig, S. Kraml, and A. Lessa, Constraining new physics with searches for long-lived particles: Implementation into SModelS, Phys. Lett. B 788, 87 (2019).

[145] G. Aad et al. (ATLAS Collaboration), Search for direct production of charginos, neutralinos and sleptons in final states with two leptons and missing transverse momentum in $p p$ collisions at $\sqrt{s}=8 \mathrm{TeV}$ with the ATLAS detector, J. High Energy Phys. 05 (2014) 071.

[146] S. Chakraborti, A. Dutta Banik, and R. Islam, Probing multicomponent extension of inert doublet model with a vector dark matter, Eur. Phys. J. C 79, 662 (2019).

[147] A. Datta, N. Ganguly, N. Khan, and S. Rakshit, Exploring collider signatures of the inert Higgs doublet model, Phys. Rev. D 95, 015017 (2017).

[148] G. Bélanger, F. Boudjema, A. Pukhov, and A. Semenov, micrOMEGAs4.1: Two dark matter candidates, Comput. Phys. Commun. 192, 322 (2015).

[149] G. Bélanger et al., micrOMEGAs5.3, http://lapth.cnrs.fr/ micromegas/.

[150] G. Belanger, A. Mjallal, and A. Pukhov, Recasting direct detection limits within micrOMEGAs and implication for non-standard dark matter scenarios, Eur. Phys. J. C 81, 239 (2021).

[151] M. Aguilar et al. (AMS Collaboration), Antiproton Flux, Antiproton-to-Proton Flux Ratio, and Properties of Elementary Particle Fluxes in Primary Cosmic Rays Measured with the Alpha Magnetic Spectrometer on the International Space Station, Phys. Rev. Lett. 117, 091103 (2016).

[152] Y. Génolini, M. Boudaud, M. Cirelli, L. Derome, J. Lavalle, D. Maurin, P. Salati, and N. Weinrich, New minimal, median, and maximal propagation models for dark matter searches with Galactic cosmic rays, Phys. Rev. D 104, 083005 (2021).

[153] M. Aguilar et al. (AMS Collaboration), Precision Measurement of the Boron to Carbon Flux Ratio in Cosmic Rays from 1.9 GV to 2.6 TV with the Alpha Magnetic Spectrometer on the International Space Station, Phys. Rev. Lett. 117, 231102 (2016).

[154] A. Reinert and M. W. Winkler, A precision search for WIMPs with charged cosmic rays, J. Cosmol. Astropart. Phys. 01 (2018) 055.

[155] A. Acharyya et al. (CTA Collaboration), Sensitivity of the Cherenkov telescope array to a dark matter signal from the galactic centre, J. Cosmol. Astropart. Phys. 01 (2021) 057. 